\title{
A comprehensive study of NGC 2345, a young open cluster with a low metallicity ${ }^{\star}$
}

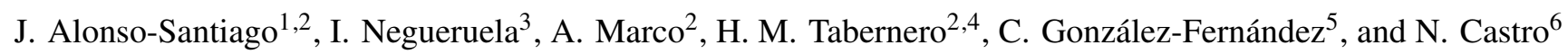 \\ 1 INAF, Osservatorio Astrofisico di Catania, Via S. Sofia 78, 95123 Catania, Italy \\ e-mail: javier.alonso@inaf.it \\ 2 Dpto de Física, Ingeniería de Sistemas y Teoría de la Señal, Escuela Politécnica Superior, Universidad de Alicante, Carretera de \\ San Vicente del Raspeig s/n, 03690, Spain \\ e-mail: javier.alonso@ua.es \\ 3 Dpto de Física Aplicada. Universidad de Alicante, Carretera de San Vicente del Raspeig s/n, 03690, Spain \\ 4 Centro de Astrobiología (CSIC-INTA), Carretera Ajalvir km 4, 28850 Torrejón de Ardoz, Madrid, Spain \\ 5 Institute of Astronomy, University of Cambridge, Madingley Road, Cambridge CB3 OHA, UK \\ ${ }^{6}$ Leibniz-Institut für Astrophysik Potsdam, An der Sternwarte 16, 14482 Potsdam, Germany
}

Received 14 June 2019 / Accepted 10 September 2019

\begin{abstract}
Context. NGC 2345 is a young open cluster that hosts seven blue and red supergiants, low metallicity, and a high fraction of Be stars, which makes it a privileged laboratory to study stellar evolution.

Aims. We aim to improve the determination of the cluster parameters and study the Be phenomenon. Our objective is also to characterise the seven evolved stars found in NGC 2345 by deriving their atmospheric parameters and chemical abundances.

Methods. We performed a complete analysis combining for the first time ubvy photometry with spectroscopy as well as the Gaia Data Release 2. We obtained spectra with classification purposes for 76 stars and high-resolution spectroscopy for an in-depth analysis of the blue and red evolved stars.

Results. We identify a new red supergiant and 145 B-type likely members within a radius of $18.7 \pm 1.2$ arcmin, which implies an initial mass, $M_{\mathrm{cl}} \approx 5200 M_{\odot}$. We find a distance of $2.5 \pm 0.2 \mathrm{kpc}$ for NGC 2345 , placing it at $R_{\mathrm{GC}}=10.2 \pm 0.2 \mathrm{kpc}$. Isochrone fitting supports an age of $56 \pm 13 \mathrm{Ma}$, implying masses around $6.5 M_{\odot}$ for the supergiants. A high fraction of Be stars $(\approx 10 \%)$ is found. From the spectral analysis we estimate an average $v_{\text {rad }}=+58.6 \pm 0.5 \mathrm{~km} \mathrm{~s}^{-1}$ and a low metallicity, $[\mathrm{Fe} / \mathrm{H}]=-0.28 \pm 0.07$, for the cluster. We also determine chemical abundances for $\mathrm{Li}, \mathrm{O}, \mathrm{Na}, \mathrm{Mg}, \mathrm{Si}, \mathrm{Ca}, \mathrm{Ti}, \mathrm{Ni}, \mathrm{Rb}, \mathrm{Y}$, and $\mathrm{Ba}$ for the evolved stars. The chemical composition of the cluster is consistent with that of the Galactic thin disc. One of the $\mathrm{K}$ supergiants, S50, is a Li-rich star, presenting an $A(\mathrm{Li}) \approx 2.1$. An overabundance of $\mathrm{Ba}$ is found, supporting the enhanced $s$-process.

Conclusions. NGC 2345 has a low metallicity for its Galactocentric distance, which is comparable to typical Large Magellanic Cloud stars. It is massive enough to serve as a test bed for theoretical evolutionary models for massive intermediate-mass stars.
\end{abstract}

Key words. open clusters and associations: individual: NGC 2345 - Hertzsprung-Russell and C-M diagrams - stars: abundances stars: fundamental parameters - stars: late-type - stars: emission-line, Be

\section{Introduction}

Stellar clusters are the naturally occurring laboratories in which stellar evolution can be studied since all of their members were formed from the same interstellar cloud. Therefore, cluster stars share distance, age, and initial composition, which is the reason for which their observed evolutionary states are strongly conditioned by their initial mass. Unlike globular clusters, where stellar evolution can be easily inferred since all of the evolutionary branches are represented, open clusters instead offer us only short snapshots because they are younger and less populated. In many cases, young open clusters do not host evolved stars or just a few and, consequently, observations do not sufficiently constrain theoretical models.

The transition between stars that explode as a supernova (SN) and those that do not, to date, still presents great uncertainty. At solar metallicity, models set this limit around $10 M_{\odot}$

\footnotetext{
* Full Tables A.1 and A.4 are only available at the CDS via anonymous ftp to cdsarc.u-strasbg.fr (130.79.128.5) or via http: //cdsarc.u-strasbg.fr/viz-bin/cat/J/A+A/631/A124
}

(Poelarends et al. 2008; Doherty et al. 2015), whereas this value could decrease down to $\approx 7 M_{\odot}$ in binary systems (Podsiadlowski et al. 2004) and low-metallicity environments (Pumo et al. 2009; Ibeling \& Heger 2013). If so, the number of SNe would be more than double what has been assumed thus far. In order to shed some light on this topic, we started an observational programme to study, in a consistent way, open clusters with ages comprised between 30 and $100 \mathrm{Ma}$. Our aim is to collect a statistically significant sample that allows us to cover the AGB and RSG mass transition. For our study, we selected those clusters that are sufficiently massive to be representative, thus those that host the largest number of evolved stars in this age range (Negueruela et al. 2017; Alonso-Santiago et al. 2019). We expect to provide experimental results that can be used to improve theoretical models and be able to better constrain the physics of the most massive intermediate-mass stars. This is the third paper in the series after those devoted to NGC 6067 (Alonso-Santiago et al. 2017) and NGC 3105 (Alonso-Santiago et al. 2018).

NGC 2345 is a poorly studied cluster in the constellation of Canis Major $\left[\alpha(2000)=07^{\mathrm{h}} 08^{\mathrm{m}} 18^{\mathrm{s}}, \delta(2000)=-13^{\circ} 11^{\prime} 36^{\prime \prime}\right.$; 
$\ell=226.58, b=-2.31]$. Moffat (1974) performed the first study of the cluster employing $U B V$ photoelectric photometry for 64 stars and slit spectra for the six brightest member stars. He found the cluster to host seven bright giants, two of which have spectral type $\mathrm{A}$ and the remaining five, type $\mathrm{K}$. He found a variable reddening across the cluster ranging, in terms of $E(B-V)$, from 0.47 to 1.16. He also estimated a distance of $1.75 \mathrm{kpc}$ and an age around $60 \mathrm{Ma}$, based on the earliest spectral type observed (around B4). In that study, by counting stars on a $V$-plate down to $V=14$, he estimated the presence of 42 members within the cluster whose diameter was set at $10.5^{\prime}$. This value slightly increased to up to $12.3^{\prime}$ (hosting 238 members) when the count was extended to fainter stars, reaching a limiting magnitude around $V=17$.

Further studies on NGC 2345 are scarce. Mathew et al. (2008) find that NGC 2345 has the highest fraction of Be stars among all of the clusters contained in their sample by looking for Be stars in open clusters and by employing slitless spectroscopy. Carraro et al. (2015) took the first and only UBVRI CCD photometry to date, covering a field of view of $20^{\prime} \times 20^{\prime}$ and reaching the 20th magnitude in the $V$ band. They determined an age for the cluster, assuming solar metallicity, in the range of $63-70 \mathrm{Ma}$ and a diameter of $7.5^{\prime}$. They placed the cluster at $3 \mathrm{kpc}$, thus doubling the Moffat (1974) value.

From high-resolution spectra, Reddy et al. (2016) provide, for the first time, stellar atmospheric parameters as well as chemical abundances for three evolved members of NGC 2345. Recently, Holanda et al. (2019) performed a more detailed chemical analysis of the all five red giants found by Moffat (1974). Both studies found a metallicity for NGC 2345 around $[\mathrm{Fe} / \mathrm{H}]=$ -0.3 , a very low value given its location within the thin disc of the Galaxy.

In this work, we present the first Strömgren photometry of NGC 2345. In addition, we collect the largest collection to date of spectra for stars in the cluster field. These spectra were taken at different resolutions with several instruments over more than six years. For the first time, we provide spectra for the blue stars, including the bright A giants that have not been studied since Moffat (1974) observed them. We characterised these blue stars by obtaining their atmospheric parameters and chemical abundances as well. Additionally, we observed the red giants at high resolution, performing a detailed spectroscopic analysis. Finally, we complement our own observations with Gaia DR2 data in order to strengthen our analysis and to be able to perform the most robust multi-approach study of this cluster so far.

\section{Observations and data}

\subsection{Photometry}

We obtained $u b v y$ Strömgren photometry using the Wide Field Camera (WFC) on the 2.5-m Isaac Newton Telescope (INT) at the El Roque de los Muchachos Observatory, located on La Palma (Canary Islands, Spain). The WFC (Ives et al. 1996) is mounted at the prime focus of the INT. It is an optical mosaic camera consisting of four thinned AR coated EEV $2 k \times 4 k$ CCDs. Each CCD has a pixel scale of $0.33^{\prime \prime}$ pixel $^{-1}$. The edge to edge limit of the mosaic is $34.2^{\prime}$, although spacing around $1^{\prime}$ is neglected in the inter-chip.

The observations were acquired during the nights of December 23-25, 2011. For each band, we used different exposure times (see Table 1) in order to avoid saturated stars as well as to cover a broad magnitude range.

Reduction was performed by using a modified version of the pipeline developed at CASU for the IPHAS survey (see
Table 1. Log of photometric observations for NGC 2345 taken at INT.

\begin{tabular}{lccc}
\hline \hline NGC 2345 & \multicolumn{2}{c}{$\begin{array}{c}\mathrm{RA}=07^{\mathrm{h}} 08^{\mathrm{m}} 18^{\mathrm{s}} \mathrm{Dec}=-13^{\circ} 11^{\prime} 36^{\prime \prime} \\
(\mathrm{J} 2000)\end{array}$} \\
\cline { 2 - 4 } Filter & \multicolumn{3}{c}{ Exposure times $(\mathrm{s})$} \\
\cline { 2 - 4 } & Short & Intermediate & Long \\
\hline$u$ & 100 & 600 & 1800 \\
$v$ & 10 & 300 & 1000 \\
$b$ & 2 & 60 & 600 \\
$y$ & 2 & 20 & 100 \\
\hline
\end{tabular}

González-Solares et al. 2008, and references therein) as it uses the same instrumentation and a similar filterset. In short, the pipeline follows the standard optical reduction cascade by performing bias and dark subtraction followed by flat correction and gain homogenisation over all four detectors.

Photometry was obtained using IMCORE, from the casutools suite $^{1}$. In brief, IMCORE performs object detection based on a robust estimation of the statistical properties of the background of the image, and it looks for connected groups of pixels above a user selectable threshold. For these detections, it obtains optimal aperture photometry along with a curve-of-growth aperture correction to homogenise the measurements. Photometric calibration was done using all of the repeated observations (both of calibration fields and target cluster) in a simplified ubercal algorithm (Padmanabhan et al. 2008).

The instrumental photometry (denoted with the subscript $i$ ) was calibrated by employing the selected standard stars observed the same nights in the clusters NGC 884 and NGC 1039 (the reference and the individual photometry for these stars are shown in Marco \& Negueruela 2013). The equations used for computing the photometric transformation, according to Crawford \& Barnes (1970), are:

$$
\begin{aligned}
& V=y_{i}+(-0.003 \pm 0.012)+(-0.04 \pm 0.05) \cdot(b-y) \\
& (b-y)=(-0.008 \pm 0.008)+(0.99 \pm 0.03) \cdot(b-y)_{i} \\
& m_{1}=(0.019 \pm 0.015)+(0.93 \pm 0.05) \cdot m_{1_{i}} \\
& +(-0.09 \pm 0.05) \cdot(b-y) \\
& c_{1}=(-0.070 \pm 0.014)+(1.049 \pm 0.012) \cdot c_{1_{i}} \\
& +(0.29 \pm 0.04) \cdot(b-y) \text {. }
\end{aligned}
$$

In total, we obtained photometry for 2153 stars, which are represented as blue circles in the first finding chart (Fig. 1). In Table A.1 we list the identification number of each star (ID), the equatorial coordinates (RA, Dec), the photometric values ( $V$, $(b-y), c_{1}$, and $\left.m_{1}\right)$ together with their uncertainties and the number of measurements $(N)$. The magnitudes and colours are the averages, weighted with the variances, of all individual measurements. The errors are expressed in terms of the standard deviation around this mean.

\subsection{Spectroscopy}

We obtained spectra in the field of NGC 2345 for 76 stars in several runs, from 2010 to 2016, with different spectrographs (see Table A.2). Our sample consists of 32 photometric cluster members, according to Moffat (1974), as well as early-type stars and supergiants in the surroundings of the cluster in order to study

\footnotetext{
1 http://casu.ast.cam.ac.uk/surveys-projects/ software-release
} 
its extent. Firstly, we selected those early-type stars previously catalogued as luminous stars (Stephenson \& Sanduleak 1971) or Be stars (Stephenson \& Sanduleak 1977). We also searched for red (super)giants according to their magnitudes and colours in 2MASS. We describe each run below.

In August 2010, during the nights of the 28 and the 29, we took 126 spectra for 74 different stars by using the AAOmega spectrograph $(\mathrm{AA} \Omega)$ for classification purposes. It is mounted on the 3.9-m Anglo-Australian Telescope (AAT) at the Australian Astronomical Observatory (AAO), on Siding Spring Mountain (Australia). This instrument is a dual-beam system, with two arms covering the full wavelength range, 3700-9500 $\mathrm{A}$, via a dichroic beam splitter with crossover at $5700 \AA$. AA $\Omega$ is an optical spectrograph that can operate in the following two modes: as a Multi-Object System (MOS) or as an Integral Field Unit (IFU). In MOS mode, the setup we used for AA $\Omega$ was fed by the Two DegreeField (2dF) fibre-positioning system, at the prime focus of the AAT. In this configuration, 392 fibres are available that cover a wide field of view of $2^{\circ}$. The red arm is equipped with the $1700 \mathrm{D}$ grating (reaching a resolving power, $R \approx 10000$ around the Ca triplet), whereas the blue arm uses the $580 \mathrm{~V}$ grating $(R=1300)$. The data reduction is performed using the automatic pipeline 2DFDR provided by the AAT as described in more detail in González-Fernández et al. (2015). The detector is a $2 k \times 4 k \mathrm{E} 2 \mathrm{~V}$ CCD.

In February 2011, from the 24 to the 27, with IDS we obtained spectra for eight blue stars with the object of performing the spectral analysis to determine their stellar atmospheric parameters as well as their chemical abundances. IDS is mounted on the 2.5-m INT at El Roque de los Muchachos Observatory, in La Palma (Spain). It is a long-slit spectrograph placed at the Cassegrain focus and is equipped with a $235-\mathrm{mm}$ focal length camera. The detector used is the default one, RED+2, a $4 k \times 2 k$ CCD red-sensitive, and a low-fringing array. We used the $\mathrm{H} 1800 \mathrm{~V}$ grating at different central wavelengths, covering the spectral range around $3800-5380 \AA$ with a $R \approx 5400$.

On November 9, 2011 we re-observed six of the stars previously observed with IDS in service mode with ISIS, with the aim of obtaining high-quality spectra to analyse. ISIS is mounted at the Cassegrain focus of the 4.2-m William Herschel Telescope (WHT) in La Palma. It is a high-efficiency, double-armed, medium-resolution spectrograph, which is capable of long-slit work up to $\approx 4^{\prime}$ length and $\approx 22^{\prime \prime}$ slit width. Due to the dichroic filters, it is possible to simultaneously observe in both arms. ISIS is equipped with two $4 k \times 2 k$ CCDs, the thinned and bluesensitive EEV12 (13.5 micron) is on the blue arm, and RED+ (15 micron) is on the red arm, which is a red-sensitive camera with almost no fringing. We used the R1200B grating centred around $4500 \AA$, which provides a $R \approx 3500$.

We used WYFFOS to collect spectra of 41 stars during the three nights of September 15-17, 2012. WYFFOS works at the prime focus of the $4.2-\mathrm{m}$ WHT. It is a multi-object fibre-fed spectrograph, containing 150 science fibres plus another ten fiducial ones. Fibres are positioned by the Autofib2 system. WYFFOS covers a wide field of 40'. We employed the R600R grating that covers the spectral range between 6200-9200 $\AA$ at a resolution of $R \approx 1700$, with the aim to identify the sources that present $\mathrm{H} \alpha$ emission. The camera (WHTWFC) is a mosaic consisiting of two thinned AR coated EEV $4 k \times 4 k$ CCDs.

Finally, we resorted to FEROS in order to observe two likely blue members and the red (super)giants at high resolution. From the analysis of the latter, which were observed in three different epochs, we performed the chemical characterisation of the cluster. FEROS is mounted on the ESO/MPG 2.2-m telescope at La Silla
Observatory (Chile). It is an échelle spectrograph and covers the wavelength range from $3500 \AA$ to $9200 \AA$, providing a resolving power of $R=48000$. The spectral range is covered in 39 orders, with small gaps between the orders appearing only at the longest wavelengths. FEROS is fed by two fibres that provide simultaneous spectra of the astrophysical target plus either sky or one of the two calibration lamps. Its detector is an EEV $2 k \times 4 k$ CCD. In a first run from May 11-13, 2011 under ESO programme 087.D603(A), we took spectra for eight stars. Then, a second run with FEROS was carried out on May 29-30, 2015 for six stars under ESO programme 095.A-9020(A). Finally, we downloaded, from the ESO archive, the spectra of five stars taken with FEROS under ESO programme 096.A-9024(A) on March 11, 2016.

Figures 1 and 2 show the stars for which we have spectra and photometry. In the first finding chart, the region covered by our photometry is marked, as well as all of the stars with spectroscopy that are spread over a wide field centred on the cluster. The second chart, instead, is limited to the central part of NGC 2345.

\subsection{Archival data}

We completed our observations with data from all-sky surveys, such as 2MASS and Gaia, inside a radius of $30^{\prime}$ around the nominal cluster centre. We only took the magnitudes of the $J H K_{S}$ from the 2MASS catalogue (Skrutskie et al. 2006) for stars that have good-quality photometry (i.e. those without any " $U$ " photometric flags in the catalogue). In addition, we also employed the Gaia DR2 data (Gaia Collaboration 2018) for those stars with sufficiently good astrometric data (i.e. a parallax error smaller than 0.5 mas).

\section{Results}

Throughout this paper we used WEBDA numbering (denoted as Star), which is the one established by Moffat (1974). For the stars that were not observed by him, we employed arbitrary and sequential numbering beginning with 1000 . When possible, we used the LS designation corresponding to stars listed in the catalogue of luminous stars in the southern Milky Way compiled by Stephenson \& Sanduleak (1971). The designation in our photometry (ID) can be found in Tables A.1 and A.4. Table A.2 lists both designations for stars with spectra.

\subsection{Spectral classification}

We performed spectral classification of the observed objects based on the classical criteria detailed below. The results obtained are described depending on the temperature range, that is, hot (or blue) and cool (red) stars. For all of the stars forming our sample, Table A. 2 shows their numbering, assigned spectral types, and spectrographs with which they were observed. For three stars, because of the poor quality of their spectra, it was impossible to assign a spectral type. For all of the rest, we estimate a typical uncertainty of around one spectral subtype for our classification.

\subsubsection{Blue stars}

We took spectra from the blue stars in the field of NGC 2345 to study the upper main sequence (MS) and find which is the spectral type corresponding to the main sequence turnoff (MSTO) point in the photometric diagram. We have four highand 14 intermediate-resolution spectra for nine different stars, 


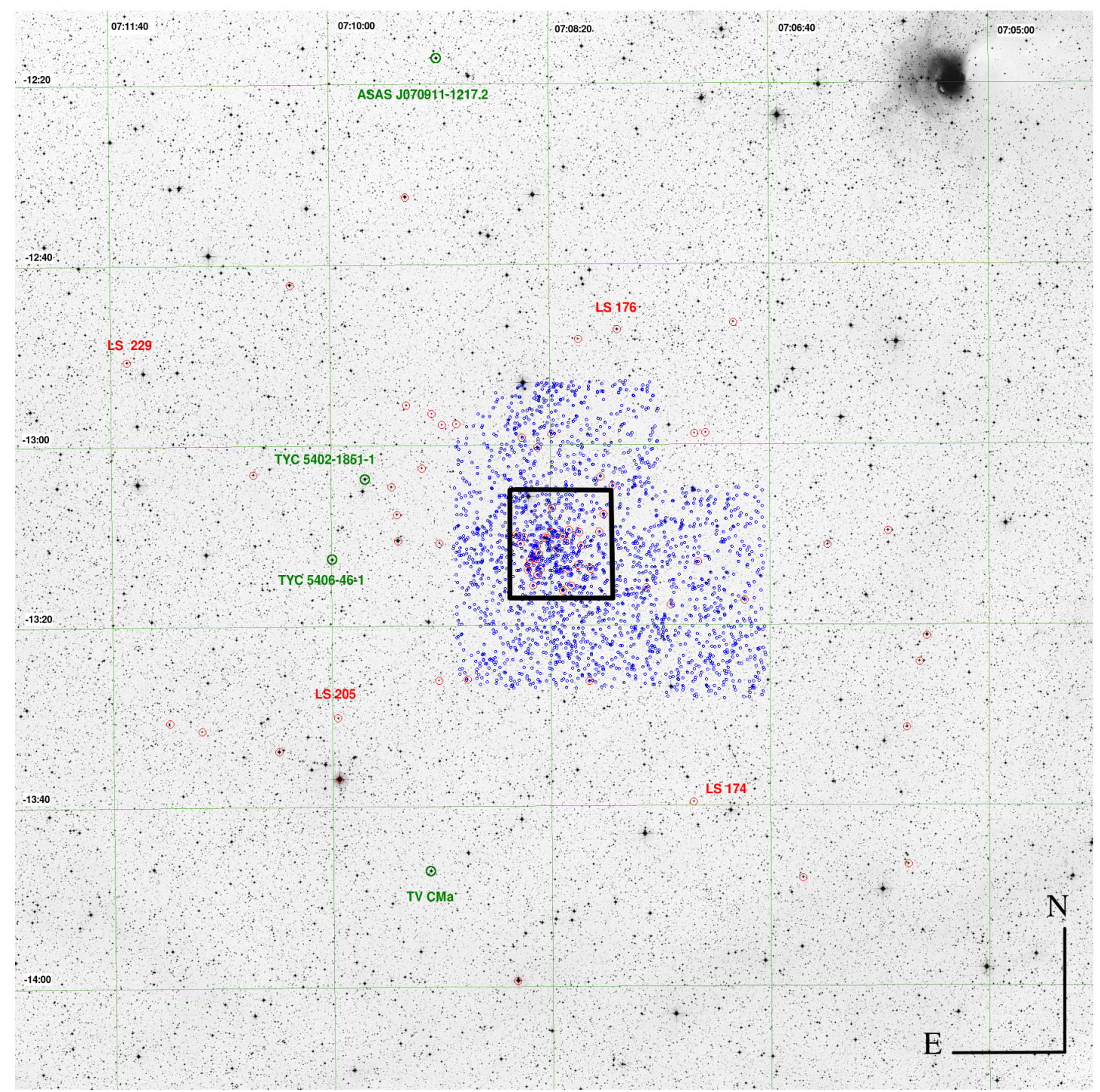

Fig. 1. Finding chart for stars with spectra (red circles) in field and surroundings of NGC 2345 . The chart is a $120^{\prime} \times 120^{\prime}$ POSS2 Red image. The stars covered by our photometry are identified as blue circles. Some stars whose relationship with the cluster is discussed in the text are also represented (green circles). The central part of the cluster, inside the black square, is shown in more detail in Fig. 2. North is up and east is on the left.

out of which two are A supergiants and seven other stars with early-mid B spectral types (see Fig. 3). We followed classical criteria for classification in the optical wavelength range (4000-5000 ^), following Jaschek \& Jaschek (1987) and Gray \& Corbally (2009).

With respect to the A supergiants around spectral type A2-A3, the Ca II K-line is a notable feature. Whereas, the profile of the Balmer lines is the following primary luminosity criterion: the narrower their wings are, the higher the luminosity. The Fe II line at $4233 \AA$, the blends of Fe II and Ti II at $4172-8 \AA$, and the Si II $24128-30$ doublet are enhanced in the supergiants.

Regarding the B-type stars, as we move toward later spectral types, the ratio $\mathrm{Mg}$ II $\lambda 4481 / \mathrm{He} \mathrm{I} \lambda 4471$ increases, as He I lines weaken. Our sample consists of stars with spectral types between $\mathrm{B} 3$ and $\mathrm{B} 4$. The line ratios used in this range to assign spectral types are Si II $\lambda 4128-30 / \mathrm{Si}$ III $\lambda 4553$, Si II $\lambda 4128-30 /$ He I $\lambda 4121$, N II $\lambda 3995 / \mathrm{He}$ I $\lambda 4009$, and He I $\lambda 4121 / \mathrm{He}$ I $\lambda 4144$ (Walborn \& Fitzpatrick 1990). Among likely members (see Table A.2), the earliest spectral type found on the main sequence is $\mathrm{B} 3 \mathrm{~V}$.
Finally, we observed at low resolution, just for classification purposes, one O-, 40 B-, and 12 A-type stars. Most of these objects are field stars located in the surroundings of the cluster in the region that is not covered by our photometry (Fig. 1).

\subsubsection{Be stars}

We identified 17 Be stars in a wide field centred on NGC 2345. Most of them (ten) are located in the central part of the cluster (i.e. $d<5^{\prime}$ from the nominal centre). We observed almost the same stars at a similar resolution, $R \approx 1500$, in two different epochs separated roughly by two years. In 2010, we took spectra around $\mathrm{H} \beta$ with $\mathrm{AA} \Omega$, whereas in 2012 we focused on $\mathrm{H} \alpha$ with WYFFOS. Eleven of these 17 Be stars showed emission in both epochs, whereas star LS 171 only showed emission in $\mathrm{H} \alpha$ in 2012. The remaining five objects were only observed once. Table 2 lists the Be stars found in this work together with their spectral types and distances from the cluster centre. In addition, for S35 we also have spectra in two epochs during 2011 around $\mathrm{H} \beta$ (taken with IDS and ISIS) in which emission was observed. 


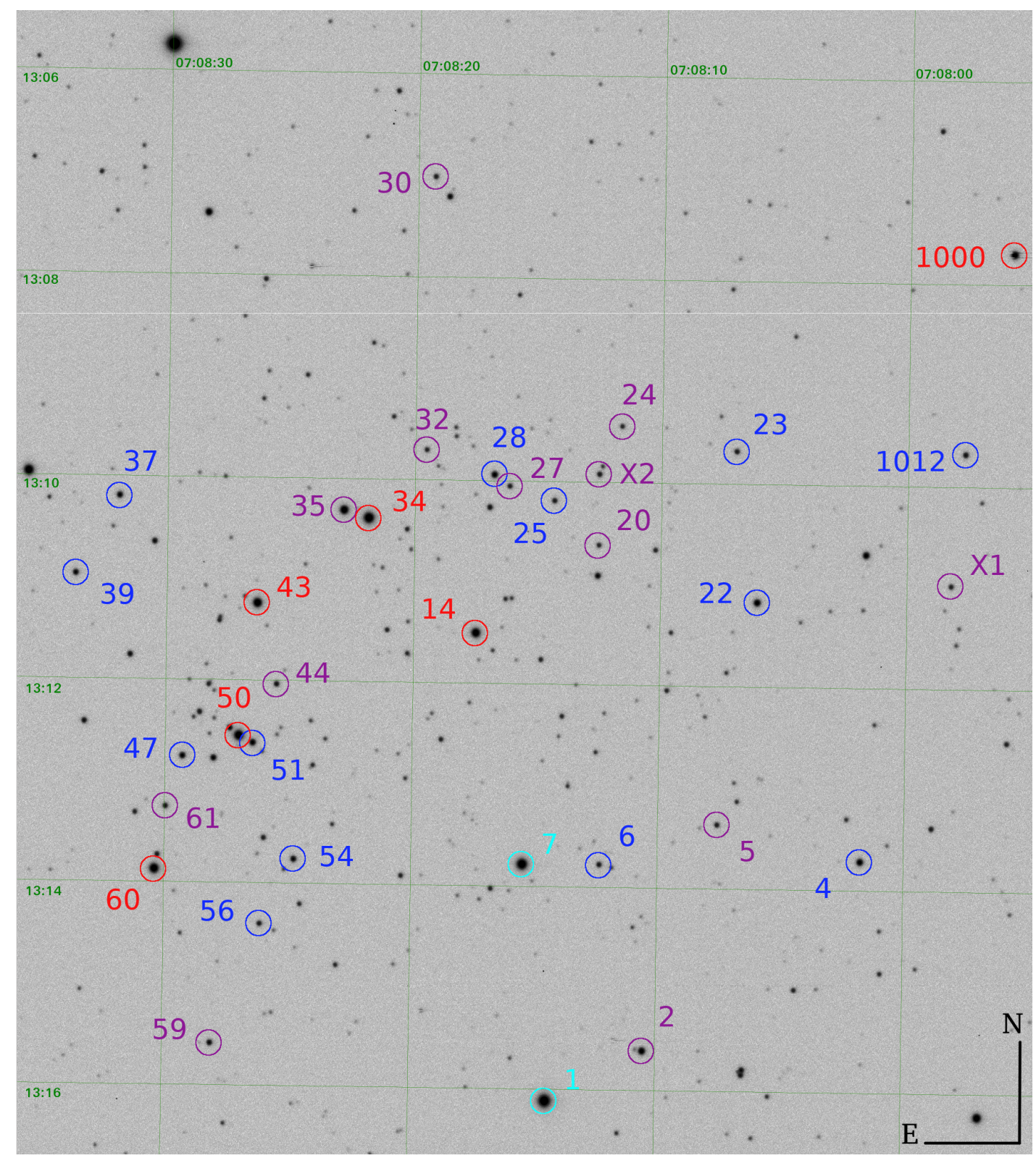

Fig. 2. Finding chart of core of NGC 2345, black square in Fig 1 . The image is one of our $V$-band frames and covers a region of sky around $10^{\prime} \times 11^{\prime}$. Stars for which we have spectra are inside circles of different colours according to their spectral types, as explained in the text, to facilitate its identification. Stars X1 and X2, identified as Be stars by Mathew \& Subramaniam (2011), without WEBDA numbering, are also included. North is up and east is on the left.

For some of these Be stars, spectra in the blue region are represented in Fig. 4. It is important to note the strong emission of stars 1003 and 1009, especially the latter, which also exhibits $\mathrm{H} \gamma$ in emission. These two stars, together with $\mathrm{S} 2$ (also mentioned in Mermilliod 1982a) and S1125, are known Be stars included in the catalogue of Galactic emission stars by Stephenson \& Sanduleak (1977). S1125 is the only one among them without any emission feature in our spectra. Although it has only been observed around $\mathrm{H} \beta$, it could still show emission in $\mathrm{H} \alpha$. In Moffat (1974), the presence of any Be star in the cluster was not mentioned. Mathew et al. (2008) carried out a survey to identify likely Be stars in 207 young open clusters using slitless spectroscopy. Later, Mathew \& Subramaniam (2011) observed and confirmed at low resolution, around $\mathrm{H} \alpha$ and $\mathrm{H} \beta$, those objects found in the previous work as Be-star candidates. In NGC 2345, they identified 12 Be stars, ten out of which have WEBDA numbering (included in Table 2). We have nine objects in common with them (only S27 was not observed by us), whereas we found emission in S30 and S63 (not quoted in their work). Two other Be stars found by Mathew \& Subramaniam (2011) without WEBDA numbering are not included in our spectroscopic sample (both are identified in the finding chart, Fig. 2).

\subsubsection{Red stars}

We took high-resolution spectra for seven evolved stars as well as low-resolution spectra for other four FG stars in the cluster field. For classification purposes, we focused on the spectral region around the $\mathrm{Ca}$ II triplet in the near-infrared wavelengths (8480-8750 A). The triplet weakens towards later spectral types and lower luminosity classes (Jaschek \& Jaschek 1987), but many 


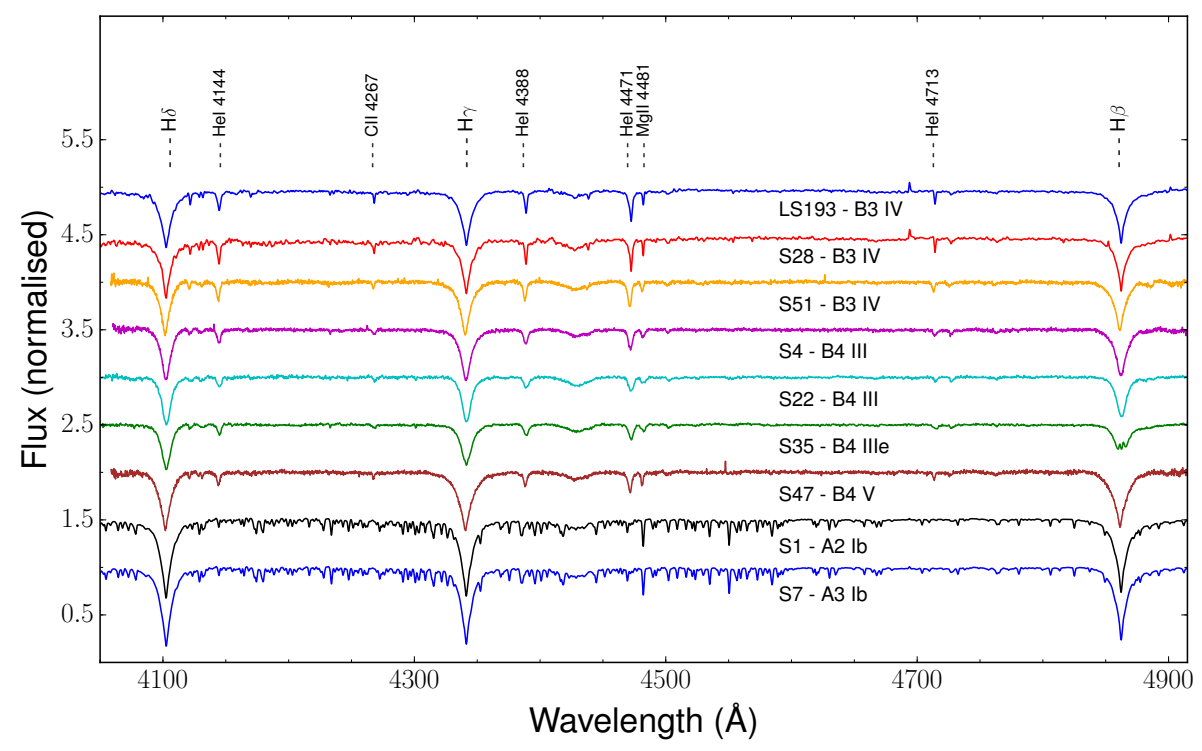

Fig. 3. Spectra of brigthest blue stars in field of cluster, taken with ISIS (Stars 4, 47, and 51), IDS (22 and 35) and FEROS (1, 7, 28, and LS193). The most important lines in this spectral region are marked. It is important to note the increasing width of the Balmer lines as we move from giants to main sequence among the mid-B stars.
Table 2. Spectral types and distances to cluster centre for Be stars found in field of NGC 2345 in this work in $\mathrm{H} \alpha$ (2012) and $\mathrm{H} \beta$ (2010).

\begin{tabular}{|c|c|c|c|c|c|c|}
\hline \multirow[t]{2}{*}{ Star } & \multirow[t]{2}{*}{$\mathrm{Sp} \mathrm{T}$} & \multirow[t]{2}{*}{$d\left(^{\prime}\right)$} & \multicolumn{2}{|c|}{ This work } & \multirow[t]{2}{*}{ Mat11 } & \multirow[t]{2}{*}{ SS } \\
\hline & & & $\mathrm{H} \alpha$ & $\mathrm{H} \beta$ & & \\
\hline \multicolumn{7}{|c|}{ Members } \\
\hline 2 & B5 IIIe & 4.4 & + & + & + & 87 \\
\hline 5 & B7 Ve & 3.1 & & + & + & \\
\hline 20 & $\mathrm{~B} 5 \mathrm{Ve}$ & 1.7 & & + & + & \\
\hline 24 & B3 IIIe & 2.6 & + & + & + & \\
\hline 27 & - & 1.6 & & & + & \\
\hline 30 & B7 IIIe & 4.6 & + & + & & \\
\hline 32 & B5 Ve & 1.9 & & + & + & \\
\hline 35 & B4 IIIe & 1.7 & + & + & + & \\
\hline 44 & B5 Ve & 1.9 & + & + & + & \\
\hline 59 & $\mathrm{~B} 5 \mathrm{Ve}$ & 4.7 & & + & + & \\
\hline 61 & B7 Ve & 3.3 & + & + & + & \\
\hline 63 & $\mathrm{~B} 5 \mathrm{Ve}$ & 12.8 & + & + & & \\
\hline 1003 & B5 Ve & 9.5 & + & + & & 86 \\
\hline \multicolumn{7}{|c|}{ Halo members } \\
\hline LS 174 & $\mathrm{~B} 2 \mathrm{Ve}$ & 31.8 & + & + & & \\
\hline LS 176 & B3 IIIe & 25.3 & + & + & & \\
\hline \multicolumn{7}{|c|}{ Non members } \\
\hline 1009 & B0.5e & 37.0 & + & + & & 82 \\
\hline 1051 & B5 III shell & 47.9 & & + & & \\
\hline 1125 & B5 III & 42.3 & & - & & 81 \\
\hline LS 171 & $\mathrm{~B} 1.5 \mathrm{Ve}$ & 32.0 & + & - & & \\
\hline
\end{tabular}

Notes. The Be stars, identified by Mathew \& Subramaniam (2011), Mat11, are added for comparison as well as those listed with their numbering (SS) according to Stephenson \& Sanduleak (1977). The plus symbol indicates emission whereas a hyphen represents the lack of it.

other classification criteria are available in this range. Many features of Fe I (i.e. lines at 8514, 8621, and $8688 \AA$ ) and Ti I (8518 become stronger with later spectral types (Carquillat et al. 1997). In addition, we find criteria for the ratios Ti I $\lambda 8518 / \mathrm{Fe}$ I $\lambda 8514$ and Ti I $\lambda 8734$ / Mg I $\lambda 8736$, which become larger with increasing spectral type, to be very useful for classification.

Figure 5 displays spectra of the evolved stars observed at high resolution, which are classified as $\mathrm{G}$ (1), K (5), and M

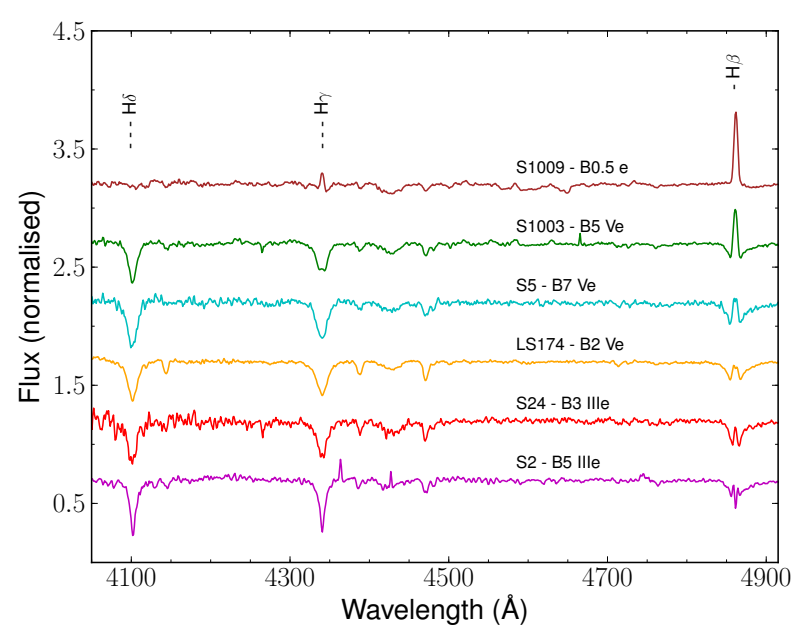

Fig. 4. AA $\Omega$ spectra of some Be stars in field of NGC 2345. As a reference, Balmer lines are marked. Stars 1009 (the only non member in this figure) and 1003 stand out especially for their strong emission. In the former star the emission phenomenon is also observable in $\mathrm{H} \gamma$.

(1) bright giants and low-brightness supergiants. Star 34 is a binary star composed of a red giant (G6 II), which dominates the composite spectrum and a blue companion (B:), which is only slightly noticeable by the weak exhibition of its Balmer lines. Besides the five RSGs found in this cluster by previous authors (Moffat 1974; Mermilliod et al. 2008; Holanda et al. 2019), we identify the following two possible candidates to be included among the cluster members: S1000 (a K supergiant) and S1002 (a M-type object). The latter is detectable in the cluster halo and thus is not covered by our photometry. Both stars (IRAS sources 07055-1302 and 07068-1321, respectively) were targeted based on their positions on the 2MASS CMD and their $\mathrm{RVs}$, obtained with $\mathrm{AA} \Omega$ in our first run, which is compatible with those of the other evolved members.

\subsection{Radial and rotational velocities}

We determined heliocentric radial velocity (RV) for the spectroscopically observed stars through Fourier cross-correlation by employing different software depending on the type of stars. For blue ones, we used the FXCOR task included in the IRAF 


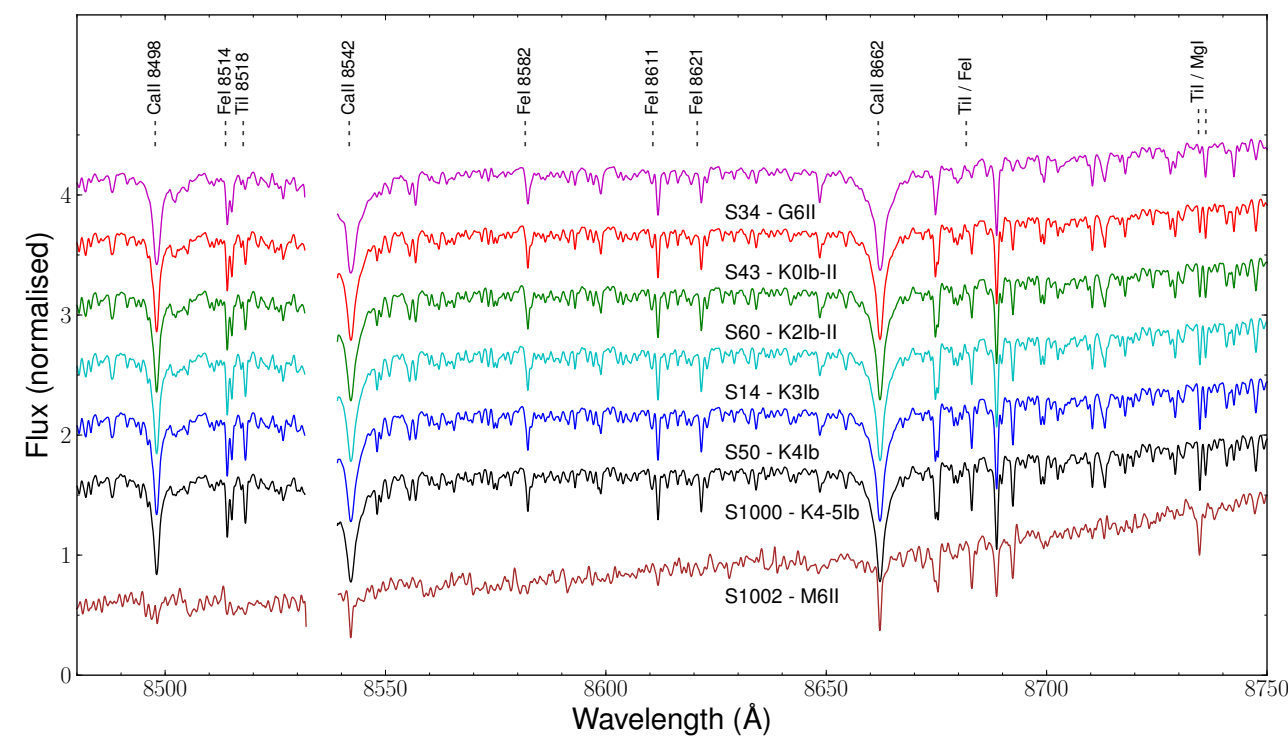

Fig. 5. FEROS spectra of the red supergiants observed around the Ca II triplet. It is important to note the gap between orders 37 and 38 between $8532-8540 \AA$. $\mathrm{S} 1000$ is the new $\mathrm{K}$ supergiant found in this work. package ${ }^{2}$. We correlated the spectrum of each star against a template and the correlation peak was fitted with a Gaussian. The templates were selected among known stars covering the B spectral type from a previous work (Alonso-Santiago et al. 2017). We focused our attention on the range between 4000 and $5000 \AA$. In order to improve the correlation, the diffuse interstellar band around $4428 \AA$ was masked as well as the region around $\mathrm{H} \beta$ when emission is strong. Conversely, in the case of cool stars, we employed ISPEC (Blanco-Cuaresma et al. 2014) and computed the cross-correlation against a list of atomic lines masks, carefully selected for the Gaia benchmark stars library pipeline, from asteroids observed with the NARVAL spectrograph.

For objects with moderate- (IDS) and high-resolution (FEROS) spectra, we determine accurate RVs displayed in Tables 3 and 4. For the remaining stars, their RVs derived from the AA $\Omega$ spectra are listed in Table A.2. Most of them correspond to blue stars that have only been observed with this instrument. Since the resolution used is low $(R=1300)$, our errors in the cross-correlation are of the order of $30 \mathrm{~km} \mathrm{~s}^{-1}$ (around $10 \%$ of instrumental broadening). For this reason the RV listed for these stars should only be considered as an indicative value of their real RV.

From red giants (i.e. S14, S43, S50, and S60), excluding S34 because of its binarity, we estimated the mean RV for the cluster by averaging the individual values, obtaining a $v_{\text {rad }}=+58.5 \pm$ $0.5 \mathrm{~km} \mathrm{~s}^{-1}$, where the error is the dispersion between the values for the different stars. In the same way Mermilliod et al. (2008) and Holanda et al. (2019) computed similar RVs: $59.2 \pm 0.7 \mathrm{~km} \mathrm{~s}^{-1}$ and $58.5 \pm 0.4 \mathrm{~km} \mathrm{~s}^{-1}$, respectively. Instead, Reddy et al. (2016) used stars 34,43 , and 60 , deriving a $v_{\mathrm{rad}}=59.7 \pm 3.4 \mathrm{~km} \mathrm{~s}^{-1}$. The large dispersion is produced by star 34 , a binary object whose RV is somewhat larger than the rest. If we do the same, we obtain a value in fair agreement with that, $60.1 \pm 3.5 \mathrm{~km} \mathrm{~s}^{-1}$.

Based on the average RV for the cluster, we selected, as likely members, stars whose RV is compatible with the mean value. All of the stars observed with FEROS, according to their RVs, are considered as likely members, including stars whose cluster membership had never been studied, namely S1000, S1002, and LS 193.

2 IRAF is distributed by the National Optical Astronomy Observatories, which are operated by the Association of Universities for Research in Astronomy, Inc., under the cooperative agreement with the National Science Foundation.
Only stars 28 and 34 show an RV that is somewhat different from the other stars. S34 can be explained by its binarity. The same explanation might be valid for S28 since its behaviour throughout the analysis carried out in this paper is fully compatible with membership. Among the members identified by Moffat (1974), all of them share an RV (derived from AA $\Omega$ spectra), which is compatible with the cluster average. Only S36, whose $v_{\text {rad }} \approx-6 \mathrm{~km} \mathrm{~s}^{-1}$ is clearly an outlier and, thus, we rejected its membership to the cluster. Taking into account the final selection of members (see Sect. 3.3) we updated the cluster RV by including all single members (blue and red, 12 in total) observed with FEROS and IDS. The resulting value is $v_{\text {rad }}=58.3 \pm 1.7 \mathrm{~km} \mathrm{~s}^{-1}$, where the error cannot be considered as a real estimate of the RV dispersion of the cluster. It is rather the scatter originated by the larger uncertainty when computing RV from blue stars (observed at lower resolution) instead of red (super)giants.

For 11 stars observed with FEROS, we determined projected rotational velocity $(v \sin i)$ by using the IACOB-BROAD code (Simón-Díaz \& Herrero 2014) based on the Fourier transform method, which separates rotation from other broadening mechanisms, such as the macroturbulent velocity $(\zeta)$. For hot stars, as a diagnostic, we used the line of Mg II at $4481 \AA$ while for cool stars we employed, at least, eight lines of Fe I and Ni I. In addition, for five other stars, all of them blue objects, we only have IDS spectra. Their resolution is not high enough to employ the usual method for separating different broadenings. In this case we assumed that the whole broadening has a rotational origin. Starting from a first estimation of the $v \sin i$ (around $50 \mathrm{~km} \mathrm{~s}^{-1}$, a value close to the resolution), a model capable of reproducing the observed spectrum was generated. In an iterative way, once we set the stellar parameters, we looked for a second estimate of rotation by changing its value until finding a new model which reproduced the profiles best. After repeating the process a couple of times it was found that the rotation did not change. These velocities are listed in Table 3 for blue stars and in Table 4 for the cool objects. The errors reflect the scatter between measurements, in terms of rms.

\subsection{Cluster membership}

The disentanglement and identification of cluster members from field stars is not a straightforward task. The very precise astrometry provided by Gaia DR2 is the ideal tool to carry it out. As 
Table 3. Log of FEROS and IDS spectra for blue stars and stellar atmospheric parameters derived from them.

\begin{tabular}{|c|c|c|c|c|c|c|c|c|}
\hline Star & Sp T & $t_{\exp }(\mathrm{s})$ & $S / N$ & $v_{\text {rad }}\left(\mathrm{km} \mathrm{s}^{-1}\right)$ & $v \sin i\left(\mathrm{~km} \mathrm{~s}^{-1}\right)$ & $\zeta\left(\mathrm{km} \mathrm{s}^{-1}\right)$ & $T_{\text {eff }}(\mathrm{K})$ & $\log g$ \\
\hline \multicolumn{9}{|c|}{ FEROS } \\
\hline 1 & A2 Ib & 1350 & 160 & $55.57 \pm 0.25$ & $25.3 \pm 0.2$ & $22.4 \pm 0.7$ & $8250 \pm 1000$ & $1.9 \pm 0.4$ \\
\hline 7 & A3 Ib & 1500 & 150 & $60.12 \pm 0.21$ & $24.1 \pm 1.0$ & $24.7 \pm 3.3$ & $8250 \pm 1000$ & $2.0 \pm 0.3$ \\
\hline 28 & B3 IV & 1000 & 70 & $49.06 \pm 0.57$ & $29.7 \pm 2.7$ & $32.0 \pm 2.0$ & $16000 \pm 1400$ & $3.7 \pm 0.2$ \\
\hline LS 193 & B3 IV & 1000 & 70 & $56.96 \pm 0.61$ & $43.2 \pm 1.2$ & $15.5 \pm 3.2$ & $16000 \pm 1300$ & $3.7 \pm 0.1$ \\
\hline \multicolumn{9}{|c|}{ IDS } \\
\hline 4 & B4 III & 3600 & 80 & $62.16 \pm 0.98$ & 170 & - & $15000 \pm 1000$ & $3.3 \pm 0.1$ \\
\hline 22 & B4 III & 3600 & 80 & $56.89 \pm 1.44$ & 210 & - & $14000 \pm 1000$ & $3.0 \pm 0.1$ \\
\hline 35 & B4 IIIe & 3200 & 110 & $57.52 \pm 0.24$ & 210 & - & $15000 \pm 1000$ & $3.0 \pm 0.1$ \\
\hline 47 & B4 V & 1800 & 80 & $56.30 \pm 1.69$ & 90 & - & $15000 \pm 1000$ & $3.7 \pm 0.1$ \\
\hline 51 & B3 IV & 3600 & 80 & $57.67 \pm 2.00$ & 110 & - & $16000 \pm 1000$ & $3.5 \pm 0.1$ \\
\hline
\end{tabular}

Table 4. Number of FEROS spectra $(N)$ for cool stars and average stellar atmospheric parameters derived from them.

\begin{tabular}{lccccccccc}
\hline \hline Star & Sp T & $N$ & $S / N$ & $v_{\text {rad }}\left(\mathrm{km} \mathrm{s}^{-1}\right)$ & $v \sin i\left(\mathrm{~km} \mathrm{~s}^{-1}\right)$ & $\zeta\left(\mathrm{km} \mathrm{s}^{-1}\right)$ & $T_{\text {eff }}(\mathrm{K})$ & $\log g$ & {$[\mathrm{Fe} / \mathrm{H}]$} \\
\hline 14 & K3 Ib & 3 & 82 & $58.92 \pm 0.12$ & $4.9 \pm 1.3$ & $4.83 \pm 0.26$ & $4014 \pm 47$ & $0.72 \pm 0.13$ & $-0.33 \pm 0.06$ \\
$344^{(*)}$ & G6 II & 2 & 140 & $64.16 \pm 0.13$ & $8.1 \pm 1.7$ & $10.10 \pm 0.22$ & $4801 \pm 50$ & $1.02 \pm 0.14$ & $-0.19 \pm 0.05$ \\
43 & K0 Ib-II & 2 & 102 & $58.34 \pm 0.08$ & $4.9 \pm 1.2$ & $4.93 \pm 0.44$ & $4246 \pm 25$ & $1.06 \pm 0.09$ & $-0.29 \pm 0.04$ \\
50 & K4 Ib & 3 & 83 & $59.02 \pm 0.38$ & $5.6 \pm 1.2$ & $3.85 \pm 1.12$ & $3948 \pm 45$ & $0.69 \pm 0.14$ & $-0.34 \pm 0.07$ \\
60 & K2 Ib-II & 2 & 92 & $57.85 \pm 0.02$ & $5.5 \pm 0.8$ & $5.68 \pm 0.17$ & $4183 \pm 52$ & $0.96 \pm 0.09$ & $-0.28 \pm 0.07$ \\
1000 & K4-5 Ib & 2 & 73 & $59.03 \pm 0.22$ & $5.9 \pm 1.8$ & $3.64 \pm 0.71$ & $3873 \pm 48$ & $0.66 \pm 0.17$ & $-0.25 \pm 0.09$ \\
1002 & M6 II & 1 & 60 & $59.69 \pm 0.17$ & - & - & $3000 \pm 100$ & $1.5 \pm 0.5$ & $0.10 \pm 0.25$ \\
\hline
\end{tabular}

Notes. ${ }^{(*)}$ This star exhibits a composite spectrum, whose companion is a blue star (B:).

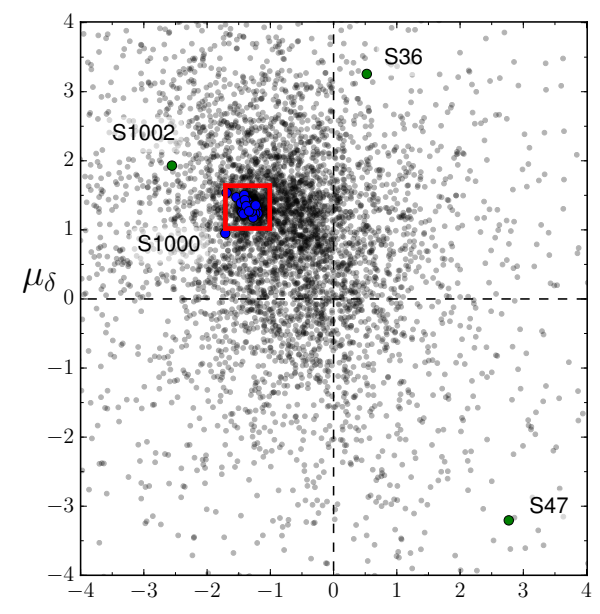

Fig. 6. Proper-motion diagram for NGC 2345. Left: grey dots are the Gaia sources within $10^{\prime}$ around the nominal cluster centre, whereas coloured circles represent stars observed spectroscopically in this work: in blue, likely members previously cited in the bibliography and in green, field stars. The red square draws the boundary of the cluster according to that described in the text. Right: for clarification purposes only, Gaia sources are represented: the cluster clearly stands out from the background. a first step, we looked for the likely cluster members by resorting to the Gaia DR2 astrometric data, that is, parallax $(\varpi)$ and proper motions $\left(\mu_{\alpha^{*}}, \mu_{\delta}\right)$. From the Gaia values for the members already known according to the literature (Moffat 1974), we placed the cluster in the space of the astrometric parameters. By employing 31 stars in the cluster core for which we have spectra (see Table A.3), we found the cluster at $\left(\varpi, \mu_{\alpha *}, \mu_{\delta}\right)_{\mathrm{cl}}=(0.35$, $-1.36,1.33) \pm(0.03,0.10,0.09)$ after discarding two field stars (S47 and S36, the latter in addition with a radial velocity totally incompatible with that of the cluster). The error $(\sigma)$ is expressed in terms of the standard deviation of the individual values. Our determination is very similar to what was computed in an automatic way by Cantat-Gaudin et al. (2018), who also used Gaia data $(0.348,-1.332,1.340)$.
Once we obtained the "cluster centre" in the astrometric space, we defined the cluster as those stars inside a circle centred at $\left(\varpi, \mu_{\alpha^{*}}, \mu_{\delta}\right)_{\mathrm{cl}}$ within a 3- $\sigma$ radius as a second step. Additionally, in order to take into account the Gaia uncertainties, we added the average astrometric errors found for the known members $(0.03,0.05,0.04)$ to this radius. In this way, we identified 834 likely cluster members within the range $0.23 \leq \varpi \leq 0.47$, $-1.71 \leq \mu_{\alpha *} \leq-1.01$, and $1.02 \leq \mu_{\delta} \leq 1.64$. In Fig. 6 the proper-motion diagram for the cluster is represented. On this diagram, the field stars S36 and S47 clearly lie outside the cluster boundary, as previously defined. In addition, the location of the two possible new (super)giants is shown. Both of them have an RV that is fully compatible with that of the cluster (Sect. 3.2); furthermore, they occupy a position on the 2MASS CMD that 


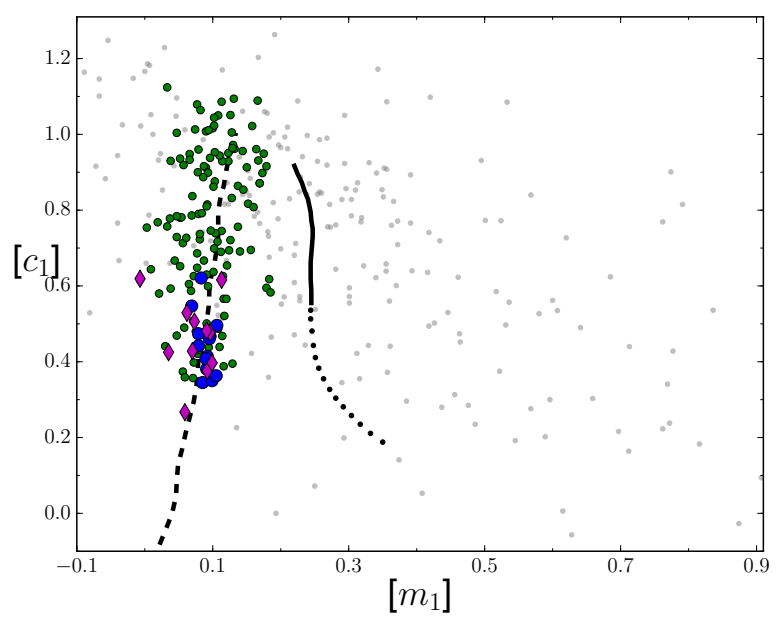

Fig. 7. $\left[c_{1}\right] /\left[m_{1}\right]$ diagram for likely members of NGC 2345 . The black lines represent the standard relation from Perry et al. (1987) for B stars (dashed line), A stars (solid line), and F stars (dotted line). B and Be stars observed spectroscopically are shown as blue circles and magenta diamonds, respectively. Green circles indicate those stars selected as Btype among all of the remaining members (grey dots).

is similar to the rest of red members. Nevertheless, according to the astrometric data we are forced to discard the membership of $S 1002$ to the cluster $(0.4178 \pm 0.1514,-2.556 \pm 0.228$, $1.936 \pm 0.196)$. Conversely, S1000 is on the cusp of being considered a member based on its astrometry $(0.3932 \pm 0.0539$, $-1.712 \pm 0.078,0.950 \pm 0.066)$. However, taking into account its astrometric uncertainties (greater than the average values), the star can be accepted as a cluster member. Additionally, we claim for its membership since from the spectroscopic analysis we do not find any significant differences with respect to the other red members already confirmed.

Finally, we have also evaluated the membership of the stars observed with AA $\Omega$ in the surroundings of the cluster. According to their RVs and, specially, their Gaia data, we find that eight stars (namely S1003, S1010, S1012, LS 174, LS 176, LS 193, LS 205, and LS 229) are compatible with the average values for the cluster. Morever, seven of these stars could be likely members when also taking into account that their spectral types are in agreement with those observed in the cluster. The only exception is S1010, whose spectral type, BN2 Ib, is not compatible with its membership. These seven stars are located in a wide range between $5^{\prime}$ and $52^{\prime}$ from the nominal cluster centre. This fact could be evidence of the existence of a large halo or of dispersed association around the cluster.

In young clusters like NGC 2345, B-type stars populate the upper MS, being the brightest cluster stars (obviously not taking into account the evolved members). These stars are key in our work since they help us estimate the reddening and the mass of the cluster. For this reason, the next task consists of identifying the B-type stars among all of the members previously found, for which the Strömgren photometry is particularly suitable. We crossed our photometry with the likely members that are astrometrically selected, and we find 365 objects in common (and 361 when crossing it with the likely members selected by CantatGaudin et al. 2018).

Then, we plotted the $\left[c_{1}\right] /\left[m_{1}\right]$ diagram $^{3}$ with the aim of searching B-type stars (Fig. 7). We confirm the reliability of our

\footnotetext{
$3\left[c_{1}\right]$ and $\left[m_{1}\right]$ are extinction-free indices computed as $\left[c_{1}\right]=c_{1}-0.20$ $(b-y)$ and $\left[m_{1}\right]=m_{1}+0.32 \cdot(b-y)$, respectively.
}

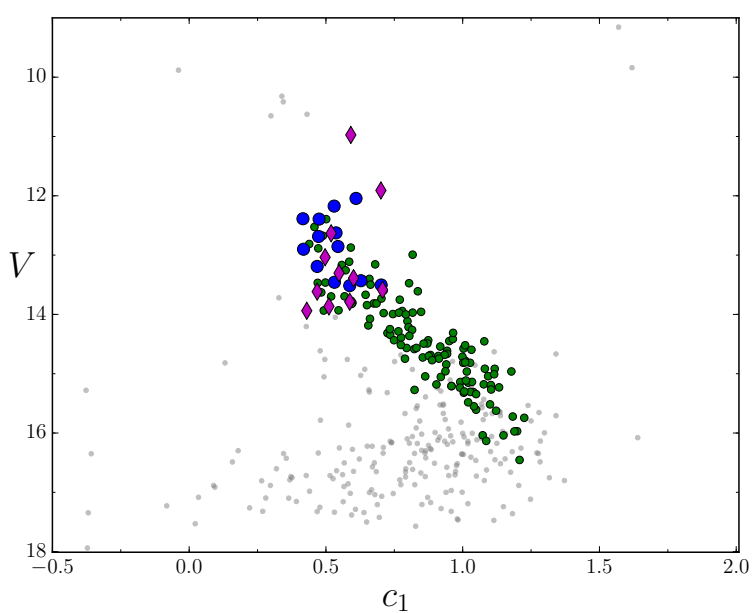

Fig. 8. $V / c_{1}$ diagram for likely members of NGC 2345 . Colours and symbols are the same as those in Fig. 7.

selection on a $V / c_{1}$ diagram, where all of these stars are located following the same sequence, as expected (Fig. 8). In order to check our analysis, we overimposed those members observed spectroscopically in both diagrams. This confirms that their location is in good agreement with their spectral types. In this way, we identified 120 B-type objects in addition to those that are already known and observed with spectra: 13 B-type and $12 \mathrm{Be}$ stars. In total, we found 145 likely B members in NGC 2345.

Finally, in order to check the impact of the field contamination in our member selection, this information is plotted in Fig. 9. We display on a Gaia CMD, the 834 likely cluster members according to their astrometric properties and, among them, we highlight those selected as B-type based on our photometric analysis. We do not appreciate an important effect of the field contamination that spoils our blue members selection. As expected, they are at the top of the cluster MS (clearly visible in the diagram), which is more than three magnitudes above the limiting value $(\approx G=20)$. Only a small dispersion is seen in their distribution, surely due to the differential reddening present across the cluster field. We note that those bright members not included in our selection are B stars that are not covered by our photometry. This also includes some LS stars such as LS 176 (which is the brightest object among the three stars with $\approx G=11$ ) and LS 195 (with a magnitude slightly fainter than $G=12$ ).

\subsection{Cluster reddening}

After evaluating the cluster membership, we estimated individual reddenings for likely B-type members by assuming standard extinction laws, that is, $E\left(c_{1}\right)=0.2 E(b-y)$ and $E(B-V)=$ $0.74 E(b-y)$. The colour excesses in $(b-y)$ were computed following the procedure described by Crawford et al. (1970) in an iterative way. We used the observed $c_{1}$ to predict the first approximation to $(b-y)_{0}$ with the expression $(b-y)_{0}=-0.116+0.097 c_{1}$. Then we calculated $E(b-y)$ and used the standard relation to correct $c_{1}$ for reddening, $c_{0}=c_{1}-E\left(c_{1}\right)$. The intrinsic colour $(b-y)_{0}$ was then calculated by replacing $c_{1}$ with $c_{0}$ in the equation above for $(b-y)_{0}$. After three iterations, we finally obtained reliable individual $E(b-y)$. From the likely members, we estimated the average cluster reddening, $E(b-y)=0.49 \pm 0.10$ corresponding to $E(B-V)=0.66 \pm 0.13$. In both cases, the errors show the standard deviation of the individual values.

The high dispersion suggests the presence of noticeable differential reddening across the observed field, as described by 


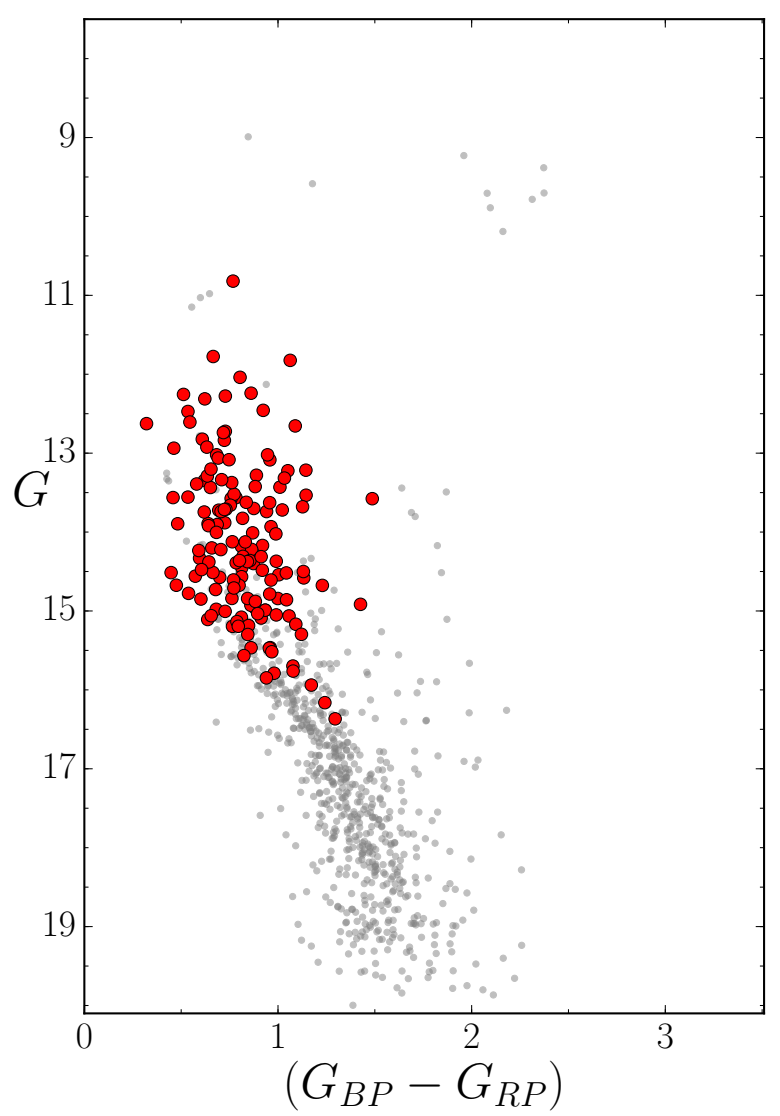

Fig. 9. $G /\left(G_{\mathrm{BP}}-G_{\mathrm{RP}}\right)$ diagram for the stars in field of NGC 2345 whose astrometry is compatible with the average cluster values. Likely B members, selected from our photometry, are shown as red circles.

previous studies (Moffat 1974; Carraro et al. 2015). We find that the reddening varies from 0.41 to 1.13 , reaching the highest values to the north-west, in the area approximately bounded by the stars 22, 23, 25, and 1012 (see Fig. 2).

\subsection{Determination of distance and age}

We continue the analysis of the cluster parameters by calculating the distance at which it is located. On the one hand, from the individual reddening for blue likely members, we carefully carried out a visual fit of these dereddened values to those calibrated by Perry et al. (1987) in the $M_{V} / c_{0}$ diagram (Fig. 10). In this way, we obtain the distance modulus, $\mu=V_{0}-M_{V}=12.0 \pm 0.2$, which corresponds to a distance of $d=2.5 \pm 0.2 \mathrm{kpc}$. The error reflects the uncertainty when visually fitting the ZAMS as a lower envelope. The combination of photometry and spectroscopy offers us the possibility of checking the validity of our result. In this way, stars for which we have spectra show absolute magnitudes according to their spectral types.

On the other hand, we check this estimate obtained from the photometric analysis with the value computed from astrometry. The cluster mean parallax derived from the 145 B likely members $(\varpi=0.349 \pm 0.039$ mas $)$ is fully compatible with that found by Cantat-Gaudin et al. (2018), 0.348, in their astrometric analysis, where they quoted a distance of $2.65 \mathrm{kpc}$. Thus, our value is consistent with the Gaia parallax. This distance places the cluster, with respect to the Galactic centre and taking as solar reference $R_{\odot}=8.3 \mathrm{kpc}$, at $R_{\mathrm{GC}}=10.2 \pm 0.2 \mathrm{kpc}$.

Once we estimated the cluster distance, we employed the isochrone fitting method to determine its age. Firstly, with the

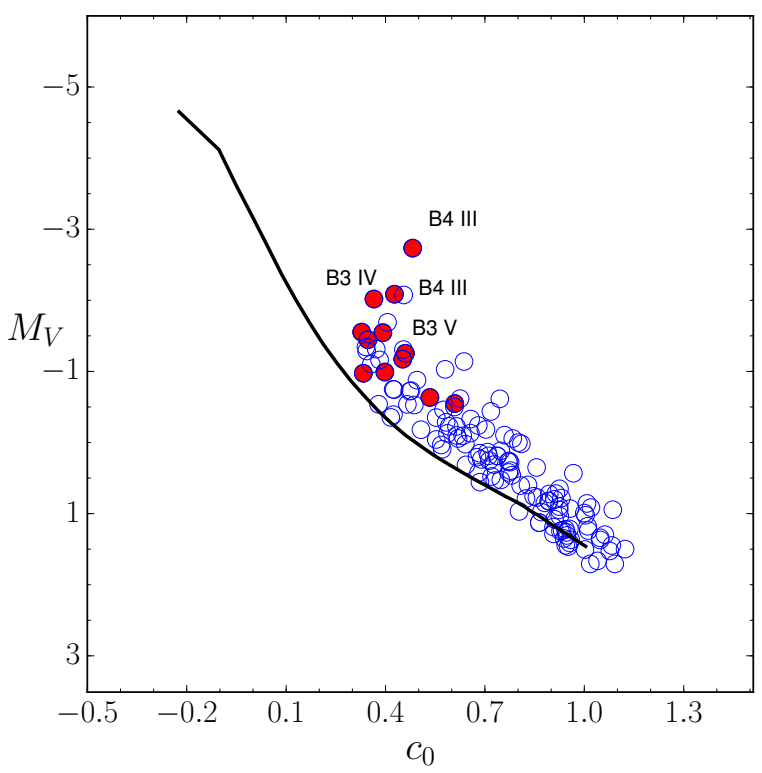

Fig. 10. $M_{V} / c_{0}$ diagram for blue likely members of NGC 2345. Stars observed spectroscopically are represented in red colour. Spectral types for the brightest stars are marked. Blue open circles are the remaining early members selected photometrically. The black line shows the calibration of Perry et al. (1987) for B stars.

intention of showing the location of the evolved stars, we plot the $V /(b-y)$ colour-magnitude diagram (CMD) for all stars in the field (Fig. 11). Before drawing the dereddened CMD on which we trace isochrones, it is necessary to deredden the remaining members, namely Be stars and blue and red (super)giants. Taking into account the differential reddening we do not use the mean value found for the cluster. Instead, we compute an individual reddening for each source by averaging those of the closest B members. The dereddened $M_{V} /(b-y)_{0} \mathrm{CMD}$ is displayed in Fig. 12 (left panel). The decrease in the stellar colour dispersion attests to the dereddening sucess.

In addition, in Fig. 12 two other CMDs are also shown: $M_{K_{S}} /\left(J-K_{S}\right)_{0}$ (central panel) and $G /\left(G_{\mathrm{BP}}-G_{\mathrm{RP}}\right)$ (right panel). In the latter case, since the dereddening of the Gaia photometry is not a trivial task, we prefer reddening the isochrone (with the corrected Gaia passbands from Maíz Apellániz \& Weiler 2018). On each CMD we plot several isochrones corresponding to different ages, shifted to the distance modulus previously obtained. The best-fitting isochrone was carefully chosen, by eye, paying special attention to the position of the evolved stars. We adopt a PARSEC isochrone (Bressan et al. 2012) with the metallicity derived for the cluster in this work, $[\mathrm{Fe} / \mathrm{H}]=-0.28$ (see Sect. 3.7.2). We converted this value into $Z$ by taking into account the aproximation $[\mathrm{M} / \mathrm{H}]=\log \left(Z / Z_{\odot}\right)$, with $Z_{\odot}=0.0152$ for PARSEC tracks.

As can be seen in Fig. 12 the 2MASS CMD yields the best fit since IR bands are less affected by the dust extinction. In this CMD, all of the evolved members lie quite well on the isochrone. Only S1000 does not match it as well as the other red giants. Morever, it is an IRAS source (numbering as 07055-1302) which suggest an IR excess. In taking this into account, the actual position of these stars could be compatible with that of an E-AGB star. In the two other CMDs, however, the fitting of the evolved stars is not good, which is probably due to the difficulty in properly correcting the variable reddening across the cluster field. The best-fitting isochrone is the same in all three CMDs, $\log \tau=7.75 \pm 0.10$, from which we estimated 


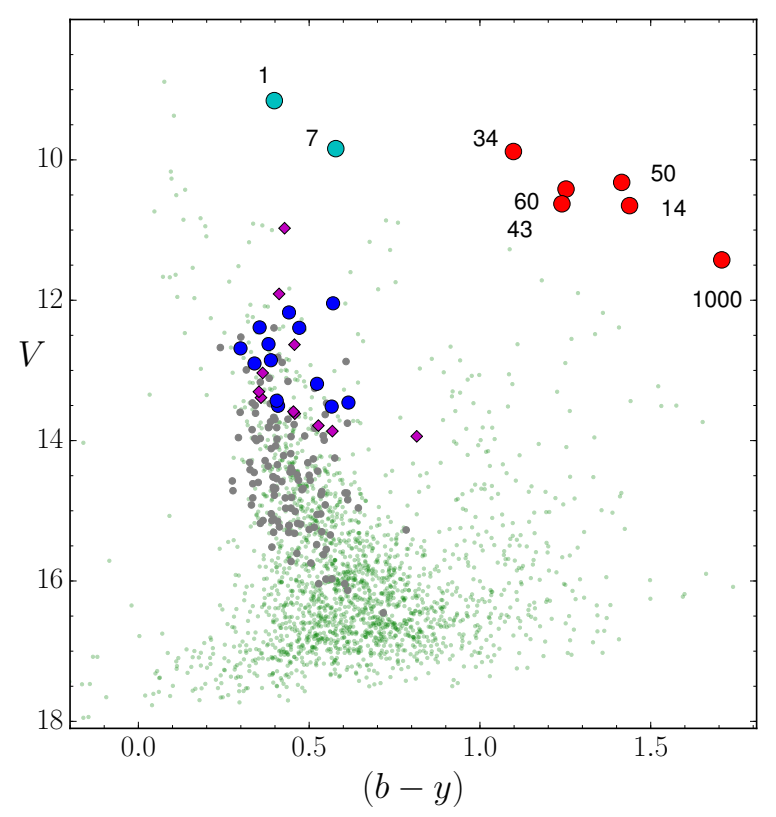

Fig. 11. $V /(b-y)$ diagram for all stars in the field of NGC 2345. Photometric data appear as green dots. Grey dots represent likely B members. Stars observed spectroscopically are represented as red circles (RSGs), cyan circles (A supergiants), blue circles (blue stars), and magenta diamonds (Be stars).

an age for the cluster of $56 \pm 13 \mathrm{Ma}$. The error shows the range of isochrones, which give a good fit. At this age, the mass of the red (super)giants corresponds to $\approx 6.5 M_{\odot}$.

\subsection{Size and mass of the cluster}

The centre of a cluster is the region where the stellar concentration is higher. By detecting this overdensity, it is therefore possible to estimate the cluster centre. For this task, we resort to the likely astrometric members previously selected. We computed the stellar density profile by counting stars along each equatorial coordinate (RA, Dec). By fitting one Gaussian to each profile, and assuming a bin size defined according to the Freedman-Diaconis rule, we find the maximum stellar density at $\alpha=107.085 \pm 0.007 \mathrm{deg}$ and $\delta=-13.197 \pm 0.008 \mathrm{deg}$. Significant differences with the nominal centre are not appreciated (this work-nominal), $\Delta \alpha=0.0 \pm 0.4 \mathrm{arcmin}$, and $\Delta \delta=0.2 \pm$ 0.5 arcmin.

We determined the cluster size from the evaluation of the stellar projected distribution around its nominal centre. The stellar density profile, $\rho(r)$, was then estimated from star counts in concentric annuli around the cluster centre up to a reasonable distance of 30 arcmin (see Fig. 13). Finally, we fitted this density profile to a three-parameters King-model (King 1962):

$\rho(r)=\rho_{0}\left\{\frac{1}{\sqrt{1+\left(r / r_{\mathrm{c}}\right)^{2}}}-\frac{1}{\sqrt{1+\left(r_{\mathrm{t}} / r_{\mathrm{c}}\right)^{2}}}\right\}^{2}$

where $\rho_{0}$ is a constant, $r_{\mathrm{c}}$ is the cluster core radius, and $r_{\mathrm{t}}$ is the tidal radius. The core radius is defined as the radial distance at which the value of density becomes half of the central density, and the tidal radius is characterised as the distance at which the cluster disappears in its environment. The values obtained from the fitting are $r_{\mathrm{c}}=3.44 \pm 0.08$ arcmin and $r_{\mathrm{t}}=18.7 \pm 1.2 \operatorname{arcmin}$, which correspond at the distance of the cluster to physical sizes of $r_{\mathrm{c}}=2.63 \pm 0.24 \mathrm{pc}$ and $r_{\mathrm{t}}=14.3 \pm 1.9 \mathrm{pc}$, respectively. This value is consistent with the position of the most remote RV members (i.e. S64, S1003, or S1020), located at $\approx 10^{\prime}$ from the cluster centre. In addition, all the B likely members are inside the cluster extent. The value calculated in this work is larger than that estimated by Piskunov et al. (2008), $r_{\mathrm{t}}=9.7 \pm 2.6 \mathrm{pc}$.

We estimate the mass of NGC 2345 following the same procedure as in the analysis of NGC 3105 (Alonso-Santiago et al. 2018, where the reader can find a detailed explanation). Once we knew the cluster size, we selected all of the B members inside this radius (in this case, including all of the early members previously selected). In order to estimate the cluster mass, we employed the multiple-part power law initial mass function (IMF) defined by Kroupa (2001). As a first step, we set the free parameter of the IMF by counting the stars within a certain mass range were we have completeness. We chose stars located at the top of the MS, from spectral type B3 V to B9 V, which amounts to 145 objects in total (previously selected in Sect. 3.3). According to the isochrone, this range of spectral types covers stars between 2.6 and $6.9 M_{\odot}$. As a second step, from this free parameter and integrating the IMF, we obtain the cluster mass. After correcting from binarity, we obtain a present cluster mass of $3700 M_{\odot}$, which is equivalent to an initial mass of $\approx 5200 M_{\odot}$. This implies that NGC 2345 is a moderately massive cluster, which is similar to other young open clusters (such as NGC 6067, NGC 3105, and Be 51).

\subsection{Stellar atmospheric parameters}

We computed stellar parameters for 16 stars following the procedures described in the analysis of NGC 3105 (Alonso-Santiago et al. 2018). We divided the sample in two different groups: the early stars and the late stars. We used, among the available spectra for each star, the one with the highest resolution. There are 11 FEROS spectra (four blue and seven cool) and five other blue objects taken with IDS. The results are displayed in Tables 3 (for blue stars) and 4 (for the cool stars).

\subsubsection{Blue stars}

We computed stellar parameters for nine blue stars from their IDS and FEROS spectra. We used a grid of FASTWIND synthetic spectra (Simón-Díaz et al. 2011; Castro et al. 2012). The stellar atmosphere code FASTWIND (Santolaya-Rey et al. 1997; Puls et al. 2005) enables non-LTE calculations and assumes a spherical geometry as well as an explicit treatment of the stellar wind effects. The technique employed was described by Castro et al. (2012), also in Lefever (2007). The stellar atmospheric parameters were derived through an automatic $\chi^{2}$-based algorithm that searches for the set of parameters that best reproduce the main strong lines observed in the range $\approx 4000-5000 \AA$. The results (i.e. effective temperature, surface gravity, and macroturbulent velocity) are shown in Table 3 . We note that the temperatures obtained are in good agreement with the values expected from the calibration by Humphreys \& McElroy (1984).

\subsubsection{Cool stars}

For the cool stars we derived stellar atmospheric parameters from their FEROS spectra. We took 1D LTE atmospheric models, specifically MARCS spherical models with $1 M_{\odot}$ (Gustafsson et al. 2008). We generated a grid of synthetic spectra by using the radiative transfer code SPECTRUM (Gray \& Corbally 1994). Although MARCS models are spherical, SPECTRUM treats them as if they are plane-parallel. Therefore, the plane parallel transfer 

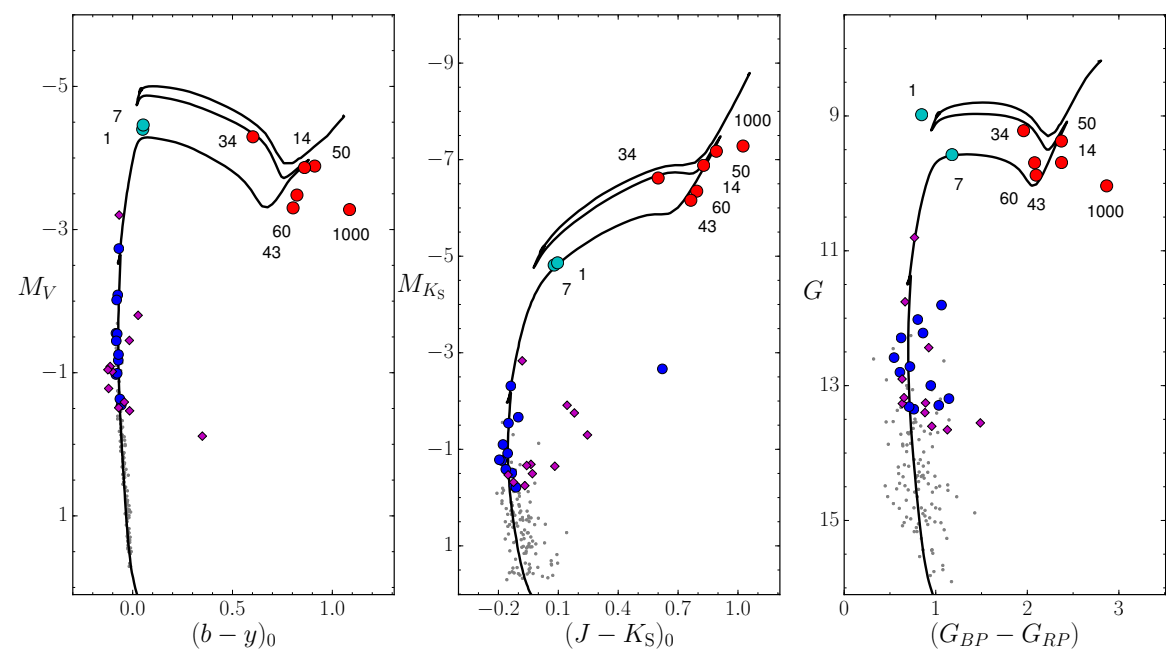

Fig. 12. Colour-magnitude diagrams for likely members of NGC 2345 in three different photometric systems: Left: $M_{V} /(b-y)_{0}$ from the Strömgren photometry taken in this work; Center: $M_{K_{S}} /\left(J-K_{S}\right)_{0}$ (2MASS photometry) and Right: $G /\left(G_{\mathrm{BP}}-G_{\mathrm{RP}}\right)$ (Gaia DR2 data). Photometric data for likely members appear as grey dots. Stars observed spectroscopically are represented as red circles (RSGs), cyan circles (A supergiants), blue circles (blue stars), and magenta diamonds (Be stars). The black line shows the best-fitting PAR$\mathrm{SEC}$ isochrone $(\log \tau=7.75)$.

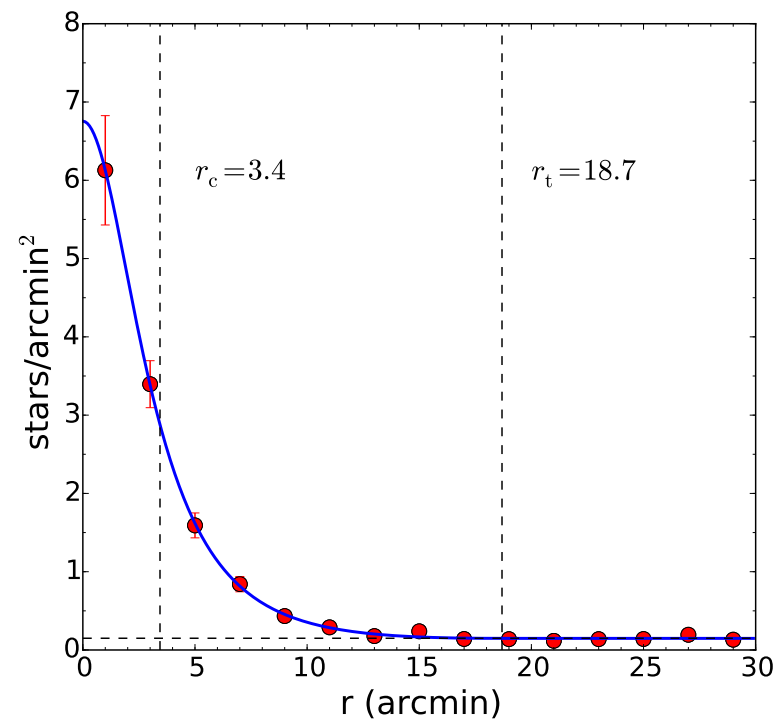

Fig. 13. Projected density distribution of likely Gaia DR2 members in cluster field. The red circles are the observed values together with their Poisson errors. The blue line shows the fitted King profile from which we obtained both the core $\left(r_{\mathrm{c}}\right)$ and the tidal $\left(r_{\mathrm{t}}\right)$ radii. Their positions are indicated with vertical dashed lines. The background density $\left(0.15\right.$ stars $\left.\operatorname{arcmin}^{-2}\right)$ is also represented as a horizontal dashed line.

treatment might produce a small inconsistency in the calculations of synthetic spectra based on MARCS atmospheric models. However, the study of Heiter \& Eriksson (2006) concluded that any difference introduced by the spherical models in a plane-parallel transport scheme is small. The microturbulent velocity $(\xi)$ was fixed according to the calibration given in Dutra-Ferreira et al. (2016). Effective temperature $\left(T_{\text {eff }}\right)$ ranges from $3300 \mathrm{~K}$ to $7500 \mathrm{~K}$ with a step of $100 \mathrm{~K}$ up to $4000 \mathrm{~K}$ and $250 \mathrm{~K}$ until $7500 \mathrm{~K}$, whereas surface gravity $(\log g$ ) varies from -0.5 dex to 3.5 dex in 0.5 dex steps. Finally, in the case of metallicity (using $[\mathrm{Fe} / \mathrm{H}]$ as a proxy), the grid covers from -1.5 dex to +1.0 dex in 0.25 dex steps.

We employed a methodology to derive stellar atmospheric parameters based on the iron linelist provided by Genovali et al. (2013) since Fe lines are numerous as well as very sensitive in cool stars. The linelist contains $\sim 230$ features for Fe I and $\sim 55$ for Fe II. Their atomic parameters were taken from the VALD database $^{4}$ (Piskunov et al. 1995; Kupka et al. 2000). For the

\footnotetext{
4 http://vald.astro.uu.se/
}

Van der Waals damping data, we took the values given by the Anstee, Barklem, and O'Mara theory, when available in VALD (see Barklem et al. 2000).

As a starting point, we employed an updated version of the STEPAR code (Tabernero et al. 2018), adapted to the present problem, that uses stellar synthesis instead of an EW method. As an optimisation method, we used the Metropolis-Hastings algorithm. Our method simultaneously generates 48 Markov-chains of 750 points for each one. It then performs a Bayesian parameter estimation by employing an implementation of Goodman \& Weare's Affine Invariant Markov chain Monte Carlo Ensemble sampler (Foreman-Mackey et al. 2013). As an objective function, we used a $\chi^{2}$ in order to fit the selected iron lines. We fixed the stellar rotation to the previously derived value, and the instrumental broadening to the resolution of the FEROS spectrograph. We left the macroturbulent broadening as a free parameter to absorb any residual broadening.

We were forced to change our methodology in the case of the M-(super)giant, S1002, since its spectrum is dominated by molecular bands that erode the continuum, thus weakening or erasing other spectral features such as most iron lines. We followed the procedure described in García-Hernández et al. (2007), focusing on the region $6670-6730 \AA$ where the TiO bands at $6681 \AA$ and $6714 \AA$ are clearly present. These bands are very useful since their depth is very sensitive to temperature (García-Hernández et al. 2007). We used the same grid of MARCS synthentic spectra but using TURBOSPECTRUM (Plez 2012) as a transfer code. For this star, we computed its stellar parameters based on a $\chi^{2}$-minimisation code implemented by us.

Results (i.e. effective temperature, surface gravity, macroturbulent velocity, and iron abundance) are displayed in Table 4. From the analysis of the six GK (super)giants, we derive a subsolar metallicity for the cluster. The weighted mean (using the variances as weights) is $[\mathrm{Fe} / \mathrm{H}]=-0.28 \pm 0.07$, quoting the uncertainty in terms of the standard deviation. Three of these cool objects, stars 14, 50, and 1000, have been observed in three different epochs separated by $\approx 5$ years. Spectral variability is not appreciated and the parameters derived from individual spectra confirm the consistency of our code. The average difference between parameters from different epochs are $\Delta T_{\text {eff }}=20 \mathrm{~K}$, $\Delta \log g=0.09 \mathrm{dex}$ and $\Delta[\mathrm{Fe} / \mathrm{H}]=0.04 \mathrm{dex}$, which are values that are rather smaller than the intrinsic errors provided by STEPAR. Once we verified this similarity, we list in Table 4 the mean parameters for each star, weighting the average by the $\mathrm{S} / \mathrm{N}$ of the individual spectrum. 
Table 5. Chemical abundances, relative to solar abundances by Grevesse et al. (2007), measured on blue stars observed with FEROS.

\begin{tabular}{lccc}
\hline \hline Star & {$[\mathrm{Si} / \mathrm{H}]$} & {$[\mathrm{Mg} / \mathrm{H}]$} & {$[\mathrm{C} / \mathrm{H}]$} \\
\hline 28 & $-0.50 \pm 0.67$ & $-0.70 \pm 0.53$ & $-0.30 \pm 0.51$ \\
LS 193 & $-0.50 \pm 0.39$ & $-0.90 \pm 0.36$ & $-0.30 \pm 0.25$ \\
\hline
\end{tabular}

\subsection{Chemical abundances}

\subsubsection{Blue stars}

We tried to derive chemical abundances for hot stars, where the main optical transitions of the chemical elements included in the stellar atmosphere grid were observed (see Castro et al. 2012). Early A-type stars fall outside the range of effective temperatures included in the FASTWIND grid (i.e. $T_{\text {eff }}<10000 \mathrm{~K}$ ). In those stars, it was not possible to perform the chemical analysis using the FASTWIND grid built in this study. We only employed highresolution spectra from FEROS since the resolution of the IDS and ISIS spectra are not the required for this sort of analysis. Finally, we only obtain abundances for two B-type stars, namely S28 and LS 193.

Once we fixed the stellar parameters, we used them to compute tailored models for each star by varying in steps of $0.2 \mathrm{dex}$ the abundances of the chemical elements under study. In these stars only the line of $\mathrm{Mg}$ II at $4481 \AA$ is strong enough to perform a good analysis. Si II and C II features are also visible but not as clear as $\mathrm{Mg}$ II. The chemical analysis is performed by employing a $\chi^{2}$ algorithm but a subsequent visual inspection is required to avoid misleading results due to weak or blended lines. The technique, as well as the lines considered in the analysis, is described in detail by Castro et al. (2012). The results are listed in Table 5 .

\subsubsection{Cool stars}

In the case of the cool stars, we computed chemical abundances for the six GK-(super)giants found in the cluster. We employed the same methodology as in the study of NGC 3105 (AlonsoSantiago et al. 2018). We used a method based on EWs measured in a semi-automatic fashion using TAME (Kang \& Lee 2012) for $\mathrm{Na}, \mathrm{Mg}, \mathrm{Si}, \mathrm{Ca}, \mathrm{Ti}, \mathrm{Ni}, \mathrm{Y}$, and $\mathrm{Ba}$. We also measured EWs by hand for two special and delicate cases, namely oxygen and lithium using the IRAF SPLOT task. For lithium, we employed a classical analysis using the $6707.8 \AA$ line, taking into account the nearby $\mathrm{Fe}$ I line at $6707.4 \AA$. We measured the EW by hand (in $\mathrm{m} \AA$ ), using the IRAF SPLOT task. We use the standard notation, where $A(\mathrm{Li})=\log [n(\mathrm{Li}) / n(\mathrm{H})]+12$. In the case of oxygen, we used the [O I] $6300 \AA$ line. This oxygen line is blended with a Ni I feature; we corrected the EWs accordingly by using the methodology and line atomic parameters described in Bertran de Lis et al. (2015). Finally we also derived rubidium abundances using stellar synthesis for the $7800 \AA \mathrm{Rb}$ I line, following the methodology in D'Orazi et al. (2013).

Table 6 summarises the abundances found for each star together with their errors. We estimated the cluster average by using a weighted arithmetic mean (employing the variances as weights). We computed conservative errors since the associated uncertainty is the combination in quadrature of the individual typical error and the star-to-star dispersion.

\section{Discussion}

In this work we perform a comprehensive study of the open cluster NGC 2345. The combination of Strömgren photometry, Gaia data, and spectroscopy at different resolutions makes our analysis, by far, the most robust of all those carried out to date.

\subsection{Cluster parameters}

Cluster parameters, such as reddening, size, distance, and age obtained for NGC 2345 by different authors are displayed in Table 7 for comparison purposes. As mentioned in Sect. 1 there are two main photometric works about this cluster. The first one (Moffat 1974) employed $U B V$ photoelectric and photographic photometry, whereas the second one (Carraro et al. 2015) performed modern CCD photometry for the first time. We place the cluster roughly at an equidistant point between previous estimates. The age obtained in this work is slightly younger, although compatible within the errors, than that given by (Carraro et al. 2015), which assumed a solar metallicity instead of a subsolar value as we did.

The number and type of supergiants contained in a cluster is an important observable to constrain the stellar evolutionary models. NGC 2345 hosts two A- and six K-type supergiants, representing a blue-to-red supergiant ratio, $B / R=0.33$, similar to what is observed in NGC $3105(B / R=0.4)$. Both values are approximately half of the expected value, $B / R=0.7$, according to the empirical expression found by Eggenberger et al. (2002). As discussed in Negueruela et al. (2018), Alonso-Santiago et al. (2018), A supergiants are mostly observed in young clusters as objects leaving the MS, and not as blue-loop objects, which contradicts the prediction of Geneva models (Georgy et al. 2013). In addition, no yellow supergiants are observed in NGC 2345 in contrast to Be 51 (Negueruela et al. 2018), a coeval cluster with a similar mass that hosts four yellow supergiants, yet none of which are blue.

\subsection{The extent of NGC 2345}

The main difference, with respect the other works, is found in the cluster size: our value for the radius is more than three times larger than that found by Moffat (1974) and five times larger in the case of the value estimated by Carraro et al. (2015). This result, obtained by using photometry, is consistent with the position of the most distant members confirmed via RV (such as LS 193 at $\approx 17^{\prime}$ ). Additionally, we have identified among the most distant objects observed with AA $\Omega$ some stars (LS 174, LS 176, LS 205, and LS 229), at distances from $25^{\prime}$ up to $50^{\prime}$, with RV and astrometric data that are compatible with those of the cluster.

Anderson et al. (2013) evaluate the membership of the Cepheids TV CMa and ASAS J070911-1217.2. Both objects are located at a great distance from the nominal cluster centre $\left(38^{\prime}\right.$ and $56^{\prime}$, respectively). In the first case, TV CMa, they discarded its relationship with NGC 2345, whereas for the second Cepheid they were not able to yield a conclusive result. In light of the new Gaia DR2 data, we directed our attention back to this issue. The proper motions and the RV $\left(37 \pm 7 \mathrm{~km} \mathrm{~s}^{-1}\right)$ are not compatible with the membership for TV CMa, as they suggest. Instead, ASAS J070911-1217.2 shows astrometric values in good agreement with the cluster average. Only the RV $\left(70 \pm 3 \mathrm{~km} \mathrm{~s}^{-1}\right)$ is somewhat different from what is expected for a member, even when taking into account that it is a variable star. In any case, it seems unlikely that this Cepheid is associated to the cluster since it is very distant and its position on the CMDs falls away from the isochrone. (see Fig. 14).

In addition, we have identified two new possible red (super)giants among all of the likely members previously selected (Sect. 3.3). These objects were neither covered by 
Table 6. Chemical abundances, relative to solar abundances by Grevesse et al. (2007), measured on cool stars.

\begin{tabular}{lcccccc}
\hline \hline Star & {$[\mathrm{O} / \mathrm{H}]$} & {$[\mathrm{Na} / \mathrm{H}]$} & {$[\mathrm{Mg} / \mathrm{H}]$} & {$[\mathrm{Si} / \mathrm{H}]$} & {$[\mathrm{Ca} / \mathrm{H}]$} & {$[\mathrm{Ti} / \mathrm{H}]$} \\
\hline 14 & $-0.21 \pm 0.11$ & $-0.19 \pm 0.36$ & $-0.14 \pm 0.06$ & $0.00 \pm 0.17$ & $-0.05 \pm 0.20$ & $-0.08 \pm 0.18$ \\
34 & $-0.18 \pm 0.09$ & $0.08 \pm 0.22$ & $-0.10 \pm 0.05$ & $0.07 \pm 0.12$ & $0.11 \pm 0.13$ & $-0.15 \pm 0.14$ \\
43 & $0.02 \pm 0.07$ & $-0.12 \pm 0.18$ & $-0.03 \pm 0.07$ & $0.08 \pm 0.10$ & $-0.08 \pm 0.14$ & $-0.19 \pm 0.16$ \\
50 & $-0.22 \pm 0.09$ & $-0.10 \pm 0.37$ & $-0.05 \pm 0.06$ & $-0.04 \pm 0.14$ & $0.07 \pm 0.17$ & $0.09 \pm 0.15$ \\
60 & $-0.04 \pm 0.09$ & $0.00 \pm 0.27$ & $-0.02 \pm 0.05$ & $0.04 \pm 0.17$ & $-0.02 \pm 0.17$ & $-0.05 \pm 0.16$ \\
1000 & $-0.19 \pm 0.11$ & $-0.15 \pm 0.41$ & $-0.11 \pm 0.11$ & $0.01 \pm 0.15$ & $0.13 \pm 0.15$ & $0.17 \pm 0.22$ \\
\hline Mean & $-0.12 \pm 0.09$ & $-0.06 \pm 0.31$ & $-0.07 \pm 0.07$ & $0.04 \pm 0.14$ & $0.03 \pm 0.16$ & $-0.15 \pm 0.17$ \\
\hline Star & {$[\mathrm{Ni} / \mathrm{H}]$} & {$[\mathrm{Rb} / \mathrm{H}]$} & {$[\mathrm{Y} / \mathrm{H}]$} & {$[\mathrm{Ba} / \mathrm{H}]$} & $\mathrm{EW}(\mathrm{Li})$ & $A(\mathrm{Li})$ \\
\hline 14 & $-0.12 \pm 0.14$ & $-0.35 \pm 0.02$ & $-0.16 \pm 0.46$ & $0.34 \pm 0.10$ & 237.1 & $1.60 \pm 0.15$ \\
34 & $-0.25 \pm 0.08$ & - & $-0.13 \pm 0.21$ & - & - & - \\
43 & $-0.15 \pm 0.08$ & - & $-0.28 \pm 0.26$ & $0.38 \pm 0.07$ & - & - \\
50 & $-0.04 \pm 0.15$ & $-0.30 \pm 0.02$ & $-0.26 \pm 0.17$ & $0.38 \pm 0.10$ & 286.7 & $2.11 \pm 0.11$ \\
60 & $-0.09 \pm 0.14$ & $-0.33 \pm 0.04$ & $-0.11 \pm 0.42$ & $0.37 \pm 0.10$ & 81.2 & $<0.58$ \\
1000 & $0.00 \pm 0.18$ & $-0.30 \pm 0.01$ & $-0.04 \pm 0.29$ & $0.43 \pm 0.09$ & 252.6 & $1.65 \pm 0.04$ \\
Mean & $-0.15 \pm 0.13$ & $-0.31 \pm 0.02$ & $-0.19 \pm 0.32$ & $0.38 \pm 0.09$ & & \\
\hline
\end{tabular}

Table 7. Summary of cluster parameters for NGC 2345 derived in this work compared to studies found in literature.

\begin{tabular}{lccccc}
\hline \hline Reference & Phot. & $E(B-V)$ & $d(\mathrm{kpc})$ & $r_{\mathrm{cl}}\left({ }^{\prime}\right)$ & Age (Ma) \\
\hline Moffat (1974) & Pg+Pe & $0.48-1.16$ & 1.75 & $5.25-6.15$ & 60 \\
Carraro et al. (2015) & CCD & $0.59 \pm 0.04$ & $3.0 \pm 0.5$ & 3.75 & $63-70$ \\
This work & CCD & $0.37-1.17$ & $2.5 \pm 0.2$ & $18.7 \pm 1.2$ & $56 \pm 13$ \\
\hline
\end{tabular}

Notes. The solar reference used for calculating Galactocentric distances is $R_{\odot}=8.3 \mathrm{kpc}$.

our photometry nor spectroscopically observed. The first one, TYC 5402-1851-1, is located at $22^{\prime}$ from the nominal cluster centre whereas the second one, TYC 5406-46-1, is $25^{\prime}$ away. Both stars share the average astrometric parameters of the cluster but their RVs are higher than the mean value $\left(68.6 \pm 0.3 \mathrm{~km} \mathrm{~s}^{-1}\right.$ and $77.0 \pm 0.2 \mathrm{~km} \mathrm{~s}^{-1}$, respectively). On the other hand, their positions on the CMDs (in contrast to the Cepheids) are close to those of other evolved stars, which could indicate a relationship with the cluster. Figure 14 shows the position of these four evolved stars on the 2MASS and Gaia CMDs.

The detection of all of these bright stars in the cluster surroundings that share the average values of the cluster, might suggest the existence of large halo spreading around NGC 2345, which is beyond the borders estimated by us in this work. These halo members are marked in Fig. 1 to illustrate their location with respect to the cluster. Likewise, their astrometric data are also displayed in Table A.3.

\subsection{Be stars}

NGC 2345 is known to host a large number of Be stars. Among the entire sample studied by Mathew et al. (2008), NGC 2345 is the cluster with the highest fraction of Be stars. Mathew et al. (2008) and Mathew \& Subramaniam (2011) identified for the first time $12 \mathrm{Be}$ stars, representing a Be fraction, that is, $\mathrm{Be} /(\mathrm{B}+\mathrm{Be})$, of $\approx 26 \%$. Instead, from our spectroscopy, among the likely members, we found 12 Be stars, nine of which are in common with them (Table 2). Without counting the B companion in S34, we also identified 14 normal B-type stars, which implies that almost half of the B stars for which we took spectra show the emission phenomenon.

According to Porter \& Rivinius (2003) classical Be stars are non-supergiant B-type stars that show or have shown Balmer lines in emission at any time. Thus, with the aim to include all of
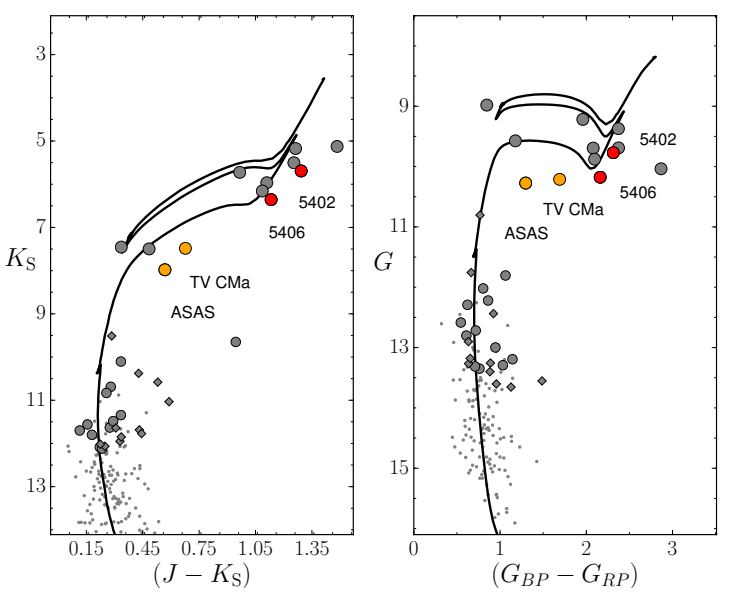

Fig. 14. 2MASS (left) and Gaia (right) colour-magnitude diagrams as in Fig. 12, but highligthing Cepheids (orange circles) and likely new red supergiants (red circles) described in text. For clarity, the names of each star have been shortened.

the Be stars in NGC 2345, we added those Be stars to our sample that were previously identified by Mathew et al. (2008) in case they were likely members. These stars, namely S27, X1, and X2, show astrometric values that are compatible with cluster membership (displayed in Table A.3) and, in fact, they had already been selected as likely B members (which proves the validity of our photometric analysis). Commonly, in young open clusters, Be stars are concentrated around the MSTO, (McSwain \& Gies 2005) as observed in NGC 2345, where they are located in the upper MS, with spectral types in the range of B3-B7 (Table 8). We found a real fraction of Be stars for NGC 2345, that is, by considering all of the likely B members and not only those observed spectroscopically, of $10.3 \%$. This value can 
Table 8. Fraction of Be stars in NGC 2345 among those members for which we have spectra.

\begin{tabular}{lccc}
\hline \hline Sp T & B & Be & Fraction $(\%)$ \\
\hline B3 & 8 & 1 & 11.1 \\
B4 & 2 & 1 & 33.3 \\
B5 & 2 & 7 & 77.8 \\
B6 & 2 & 0 & 0.0 \\
B7 & 0 & 3 & 100.0 \\
\hline Total & 14 & 12 & 46.2 \\
\hline
\end{tabular}

be considered as a lower limit since we assume that there are no more Be stars among the B members selected photometrically. Still in this case, NGC 2345 is one of the clusters with the highest Be fraction among those studied by Mathew et al. (2008).

The Be phenomenon has been observed in young open clusters hosting B stars, with ages comprised approximately between 10 and $300 \mathrm{Ma}$. Mermilliod (1982b) suggested that the Be fraction decreases with increasing age. In the Galaxy, Be stars in open clusters show a bimodal distribution peaking around spectral types B1-B2 and B7-B8 (Mermilliod 1982b) or B1-B2 and B5-B7 (Mathew et al. 2008). In NGC 2345, Be stars are concentrated around spectral type B5 and $83 \%$ are found in the bin B5-B7 (see Table 8), as it has been observed in other clusters. However, clusters with high Be fractions, such as NGC 663 (Pigulski et al. 2001), NGC 7419 (Marco \& Negueruela 2013), or NGC 3105 (Alonso-Santiago et al. 2018), seem to be grouped around ages in the range of $15-40 \mathrm{Ma}$. This is in an excellent agreement with theoretical considerations that associate the generation of circumstellar discs, responsible for the emission observed, with the rotational velocity distribution expected among early stars (Granada et al. 2016). NGC 2345 shows a high Be fraction at an atypical age (see Fig. 8 in Mathew et al. 2008).

Finally, we have to keep in mind that not only is the age key to explain the Be phenomenon but also other factors, such as the geometry of the cluster and the metallicity. On the one hand, the shape of the cluster is related to the initial angular momentum with which the cluster originated (Keller et al. 1999). Thus, a high eccentricity would be the consequence of a rapid rotation rate and, therefore, a high Be fraction would be expected. On the other hand, low-metallicity environments are known to favour the Be phenomenon (Maeder \& Meynet 2000; Martayan et al. 2007) as we argue in more detail when studying NGC 3105 (see the discussion in Alonso-Santiago et al. 2018), a younger metalpoor cluster with a comparable Be fraction.

\subsection{Atmospheric stellar parameters}

As mentioned above (Sect. 1), we found in the literature two spectroscopic studies carried out on NGC 2345 before this work. Reddy et al. (2016) derived stellar parameters and abundances only for three of the cool stars, namely 34, 43, and 60. In Table 9 we compare them with the values obtained in this work. For stars 34 and 43, our results are compatible within the incertainties, whereas for S60 all of our values are lower than theirs. More recently, Holanda et al. (2019) carried out the spectral analysis of the five red (super)giants. We reanalysed the same spectra following our procedure. Both sets of values are compatible within the errors. Only the surface gravities for S43 are significantly different (see Table 10). Regarding metallicity, our values are fully (Holanda et al. 2019) or marginally compatible (Reddy

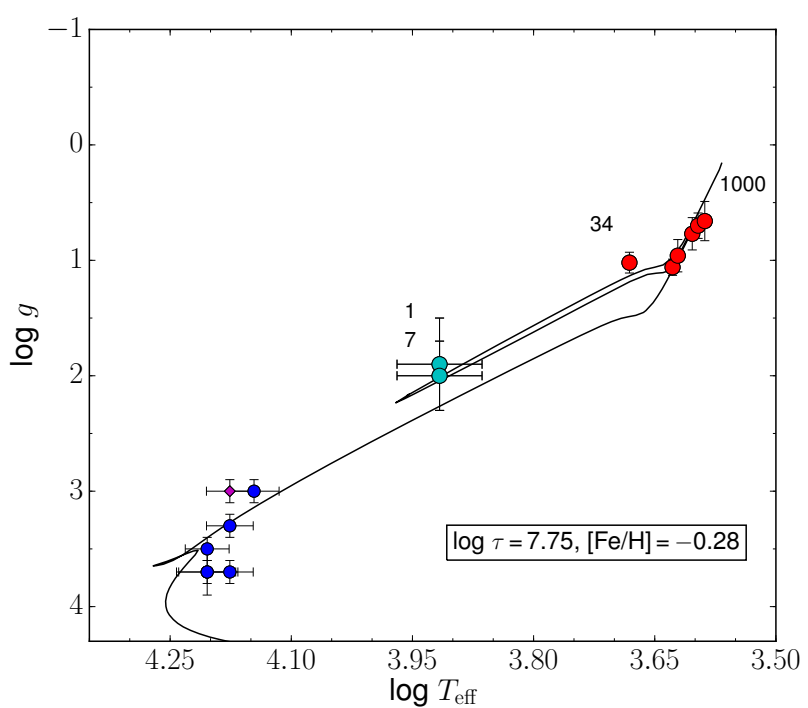

Fig. 15. Kiel diagram for likely members. Colours and symbols are the same as those in Fig. 7. The best-fitting PARSEC isochrone is the black line.

et al. 2016) with previous works, while our microturbulent velocities are somewhat smaller (on average $0.6-0.7 \mathrm{~km} \mathrm{~s}^{-1}$ ).

Finally, we plot the $\log g / T_{\text {eff }}$ (Kiel diagram) for all cluster members for which we have stellar parameters (Fig. 15). On this diagram, we also draw the best-fitting isochrone (solid line). The position of the stars matches the isochrone rather well (within the errors), especially for the evolved stars. The location of S1000 on this diagram shows an excellent agreement with the other $\mathrm{K}$ (super)giants, suggesting that its anomalous position in the photometric diagrams could be related to its intrinsic reddening, which is somewhat higher from the rest of red (super)giants. Only star 34, which is a binary, is slightly displaced from the expected evolutionary locus. This suggests that binarity may play a role in determining its evolution towards the cool side of the diagram. The excellent agreement between spectral results (stellar parameters) and cluster parameters inferred photometrically, such as the distance and age (which are reflected in the choice of the isochrone), is particularly noteworthy. This demonstrates the reliability of our results.

\subsection{Low metallicity}

We obtain a metal-poor composition for NGC 2345 of $[\mathrm{Fe} / \mathrm{H}]=$ $-0.28 \pm 0.07$. This value, computed from the six red giants, is compatible with previous estimations: $-0.28 \pm 0.05$ (Holanda et al. 2019) and $-0.17 \pm 0.06$ (Reddy et al. 2016), which used five and three stars, respectively. For a proper comparison both values have been rescaled to our solar reference (Grevesse et al. 2007).

In order to put our results in context, we place the cluster on the Galactic gradient, using the work by Genovali et al. $(2013,2014)$ as a reference. With the aim to estimate the radial distribution of metallicity, they used Cepheids since they are a young population $(\tau \approx 20-400 \mathrm{Ma})$ that trace present-day abundances. In this way, Cepheids are an appropriate subject of comparison for young clusters, such as NGC 2345. In Fig. 16, we show this gradient with the position of our cluster overplotted as well as a sample of open clusters studied by Netopil et al. (2016) for further comparison. Among them, we show young clusters (i.e. with an age below $500 \mathrm{Ma}$ ) whose metallicity is derived from, on average, at least three high-quality spectra 
Table 9. Comparison of temperature, surface gravity, microturbulent velocity, and iron abundance with those of Reddy et al. (2016).

\begin{tabular}{|c|c|c|c|c|c|c|c|c|}
\hline \multirow[t]{2}{*}{ Star } & \multicolumn{4}{|c|}{$\operatorname{Reddy} 16^{(*)}$} & \multicolumn{4}{|c|}{ This work } \\
\hline & $T_{\mathrm{eff}}(\mathrm{K})$ & $\log g$ & $\xi\left(\mathrm{km} \mathrm{s}^{-1}\right)$ & {$[\mathrm{Fe} / \mathrm{H}]^{(* *)}$} & $T_{\mathrm{eff}}(\mathrm{K})$ & $\log g$ & $\xi\left(\mathrm{km} \mathrm{s}^{-1}\right)$ & {$[\mathrm{Fe} / \mathrm{H}]$} \\
\hline 34 & 4850 & 0.85 & 3.23 & $-0.21 \pm 0.04$ & $4801 \pm 50$ & $1.02 \pm 0.14$ & 2.40 & $-0.19 \pm 0.05$ \\
\hline 43 & 4300 & 1.20 & 2.21 & $-0.19 \pm 0.04$ & $4246 \pm 25$ & $1.06 \pm 0.09$ & 1.72 & $-0.29 \pm 0.04$ \\
\hline 60 & 4300 & 1.60 & 2.21 & $-0.13 \pm 0.05$ & $4183 \pm 52$ & $0.96 \pm 0.09$ & 1.73 & $-0.28 \pm 0.07$ \\
\hline
\end{tabular}

Notes. ${ }^{(*)}$ Typical uncertainties are $\Delta T_{\text {eff }}=75 \mathrm{~K}, \Delta \log g=0.25 \mathrm{dex}$, and $\Delta \xi=0.20 \mathrm{~km} \mathrm{~s}^{-1}$. ${ }^{(*)}$ The iron abundance is rescaled to the solar reference used in this work (Grevesse et al. 2007).

Table 10. Comparison of temperature, surface gravity, microturbulent velocity, and iron abundance with those of Holanda et al. (2019).

\begin{tabular}{|c|c|c|c|c|c|c|c|c|}
\hline \multirow[t]{2}{*}{ Star } & \multicolumn{4}{|c|}{ Holanda19 ${ }^{(*)}$} & \multicolumn{4}{|c|}{ This work } \\
\hline & $T_{\text {eff }}(\mathrm{K})$ & $\log g$ & $\xi\left(\mathrm{km} \mathrm{s}^{-1}\right)$ & {$[\mathrm{Fe} / \mathrm{H}]^{(* *)}$} & $T_{\text {eff }}(\mathrm{K})$ & $\log g$ & $\xi\left(\mathrm{km} \mathrm{s}^{-1}\right)$ & {$[\mathrm{Fe} / \mathrm{H}]$} \\
\hline 14 & 4150 & 1.10 & 2.36 & -0.29 & $4024 \pm 52$ & $0.77 \pm 0.14$ & 1.71 & $-0.31 \pm 0.07$ \\
\hline 34 & 4850 & 1.10 & 3.00 & -0.20 & $4810 \pm 45$ & $1.08 \pm 0.09$ & 2.35 & $-0.19 \pm 0.05$ \\
\hline 43 & 4350 & 1.60 & 2.45 & -0.27 & $4251 \pm 21$ & $1.10 \pm 0.06$ & 1.69 & $-0.31 \pm 0.03$ \\
\hline 50 & 4000 & 0.70 & 2.31 & -0.32 & $3948 \pm 34$ & $0.70 \pm 0.10$ & 1.67 & $-0.34 \pm 0.05$ \\
\hline 60 & 4020 & 1.03 & 2.54 & -0.34 & $4200 \pm 45$ & $1.00 \pm 0.13$ & 1.72 & $-0.27 \pm 0.06$ \\
\hline
\end{tabular}

Notes. ${ }^{(*)}$ Typical uncertainties are $\Delta T_{\text {eff }}=70-100 \mathrm{~K}, \Delta \log g=0.1-0.2 \mathrm{dex}, \Delta \xi=0.20 \mathrm{~km} \mathrm{~s}^{-1}$, and $\Delta[\mathrm{Fe} / \mathrm{H}]=0.1$ dex. ${ }^{(* *)}$ The iron abundance, is rescaled to the solar reference used in this work (Grevesse et al. 2007).

(Heiter et al. 2014). The position of NGC 2345, which is very close to NGC 3105 , highlights the low metallicity of both clusters and illustrates the wide range of metallicities found in the outer region of the Galaxy. These two clusters, at a Galactocentric distances around $10 \mathrm{kpc}$, show a metallicity comparable to that of the LMC. Alonso-Santiago et al. (2017) suggest that metallicity inhibits the formation of Cepheids. Nevertheless, the absence of Cepheids in low-metallicity environments, such as NGC 2345 and NGC 3105, casts doubts on that possibility since both are massive enough clusters to serve as stellar evolution testbeds.

\subsection{Stellar chemical abundances}

NGC 2345 seems to be chemically homogeneous since for every element, abundances derived from different (cool) stars are comparable within the errors and they are all grouped around the cluster average. In Fig. 17 we show the representative star-tostar scatter by plotting the abundance of Si for all of the objects in our sample. We also derived some abundances from hot stars, but with large uncertainties, that yield a metal-poor cluster. From the analysis of both groups of stars, hot and cool, we obtained abundances marginally compatible, within the errors, for the elements in common ( $\mathrm{Si}$ and $\mathrm{Mg}$ ).

We compare the chemical abundances obtained in this work with those previously published. On the one hand, Holanda et al. (2019) derived abundances for 19 elements, nine of which are in common with those studied by us. On the other hand, Reddy et al. (2016) computed abundances for 23 species, ten of which correspond with our results. Both sets of abundances, as well as ours, are displayed in terms of the ratios $[\mathrm{X} / \mathrm{Fe}]$ in Table 11 . We computed compatible abundances, within the errors for almost all of the corresponding elements, with the exception of $\mathrm{Ca}$ and $\mathrm{Ba}$. For these elements our values are up to 0.5 dex higher.

Finally, we also compare our abundances with the Galactic trends for the thin disc (Fig. 18). We plot abundance ratios $[\mathrm{X} / \mathrm{Fe}]$ vs. $[\mathrm{Fe} / \mathrm{H}]$ obtained by Adibekyan et al. (2012) for $\mathrm{Na}$,

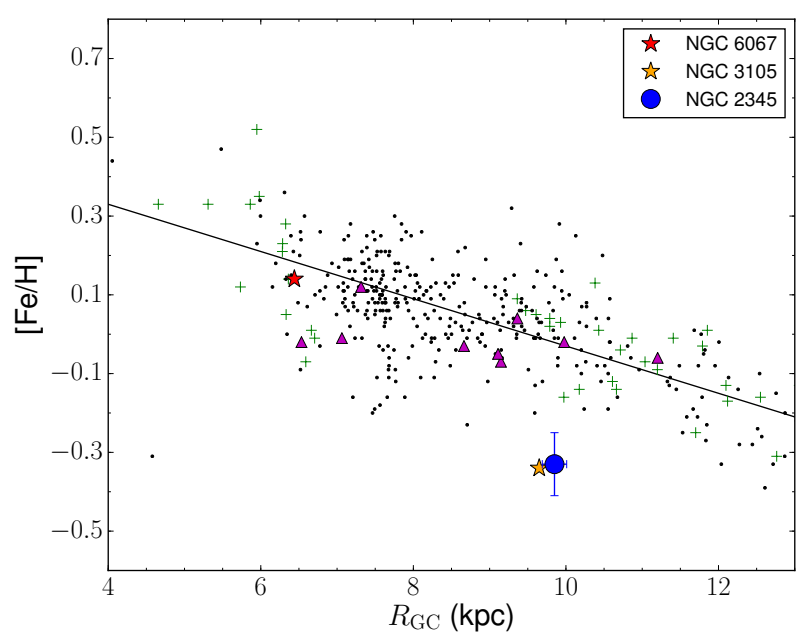

Fig. 16. Iron abundance gradient in Milky Way found by Genovali et al. $(2013,2014)$. The black line is the Galactic gradient $\left(-0.06 \mathrm{dex} \mathrm{kpc}{ }^{-1}\right)$, green crosses are Cepheids studied in those papers, whereas black dots show data for other Cepheids from literature used by these authors. Magenta triangles represent the young open clusters $(<500 \mathrm{Ma})$ in the sample compiled by Netopil et al. (2016). Finally, the blue circle is NGC 2345. NGC 6067 and NGC 3105, other young clusters analysed by our group with the same techniques, are plotted for comparison purposes. NGC 2345, together with NGC 3105, fall well below the metallicity typically observed at their. Galactocentric distance. All of the values shown in this plot are rescaled to Genovali et al. (2014), i.e. $R_{\odot}=7.95 \mathrm{kpc}$ and $A(\mathrm{Fe})=7.50$.

Mg, Si, Ca, Ti, and Ni and by Delgado Mena et al. (2017) for $\mathrm{Y}$ and $\mathrm{Ba}$ in the workframe of the HARPS GTO planet search programme. The chemical composition of NGC 2345 is compatible, within the errors, with the Galactic trends observed in the thin disc of the Galaxy. We derived a roughly solar $[\mathrm{Y} / \mathrm{Fe}]$ against a supersolar $[\mathrm{Ba} / \mathrm{Fe}]$, which is in good agreement with the dependence on age and Galactic location found 


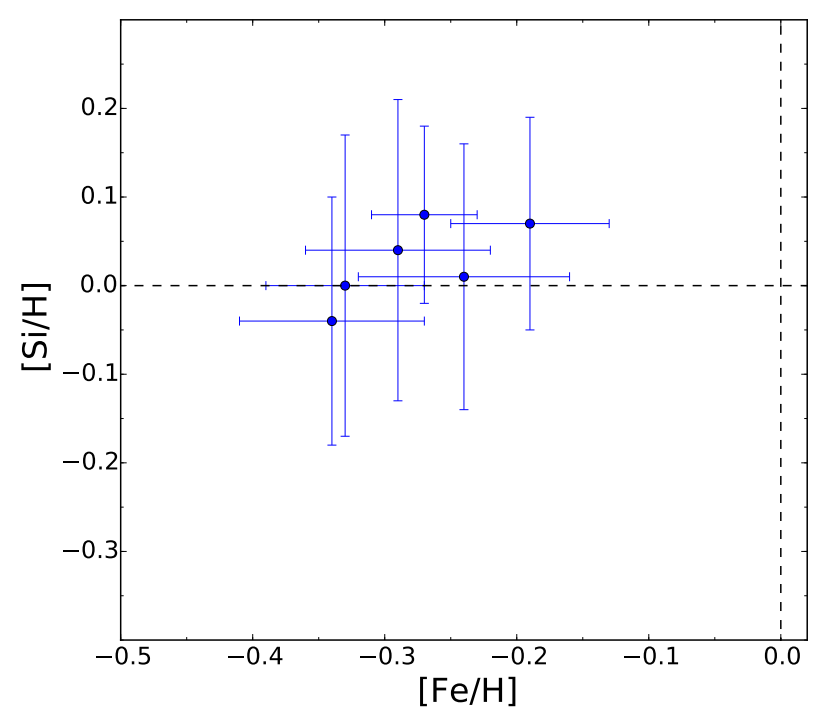

Fig. 17. Abundance ratios $[\mathrm{Si} / \mathrm{H}]$ vs. $[\mathrm{Fe} / \mathrm{H}]$. All of the stars have the same composition (within the errors), indicating chemical homogeneity. As a reference, the dashed lines show the solar values.

Table 11. Comparison of mean abundances ratios, relative to solar abundances by Grevesse et al. (2007), obtained for NGC 2345 so far.

\begin{tabular}{lccc}
\hline \hline Ratio & Reddy16 & Holanda19 & This work \\
\hline$[\mathrm{Na} / \mathrm{Fe}]$ & $+0.21 \pm 0.07$ & $+0.29 \pm 0.12$ & $+0.22 \pm 0.32$ \\
{$[\mathrm{Mg} / \mathrm{Fe}]$} & $-0.02 \pm 0.02$ & $+0.20 \pm 0.08$ & $+0.21 \pm 0.10$ \\
{$[\mathrm{Si} / \mathrm{Fe}]$} & $+0.20 \pm 0.05$ & $+0.24 \pm 0.08$ & $+0.32 \pm 0.16$ \\
{$[\mathrm{Ca} / \mathrm{Fe}]$} & $-0.25 \pm 0.10$ & $-0.02 \pm 0.03$ & $+0.31 \pm 0.17$ \\
{$[\mathrm{Ti} / \mathrm{Fe}]$} & $-0.16 \pm 0.08$ & $+0.03 \pm 0.07$ & $+0.13 \pm 0.18$ \\
{$[\mathrm{Fe} / \mathrm{H}]$} & $-0.15 \pm 0.06$ & $-0.33 \pm 0.05$ & $-0.28 \pm 0.07$ \\
{$[\mathrm{Ni} / \mathrm{Fe}]$} & $-0.15 \pm 0.06$ & $-0.12 \pm 0.01$ & $+0.13 \pm 0.15$ \\
{$[\mathrm{Y} / \mathrm{Fe}]$} & $+0.05 \pm 0.05$ & $+0.19 \pm 0.06$ & $+0.09 \pm 0.33$ \\
{$[\mathrm{Ba} / \mathrm{Fe}]$} & $+0.14 \pm 0.04$ & & $+0.66 \pm 0.11$ \\
\hline
\end{tabular}

by Mishenina et al. (2013) by comparing the abundances of $\mathrm{Y}$ and $\mathrm{Ba}$ in different open clusters. Remarkably, we find a strong over-abundance of Barium $([\mathrm{Ba} / \mathrm{Fe}]=+0.8)$. Several young open clusters have been observed to have $\mathrm{Ba}$ abundances higher than those predicted by standard theoretical models. To explain this enrichment of Ba, D'Orazi et al. (2009) suggest an enhanced "s-process". Our result supports this idea (see their Fig. 2).

\subsection{Lithium-rich stars}

Canonical models (Iben 1967a,b; Soderblom et al. 1993) predict $\mathrm{Li}$ depletion once stars reach the red giant branch as a direct consequence of the first dredge-up. According to this, we do not expect to find in red giants abundances of $\mathrm{Li}$ above 1.5 dex (Charbonnel \& Balachandran 2000). In good agreement with this scenario, $\mathrm{Li}$ is either not found or just in a small amount in stars 34, 43, and 60. Nevertheless, as in the case of NGC 6067 (Alonso-Santiago et al. 2017), we find three Li-rich stars: S14, S50, and S1000. For stars S14 and S1000, the abundance of $\mathrm{Li}(1.67 \pm 0.13$ and $1.65 \pm 0.04$, respectively) could be marginally compatible with the canonical scenario, but not for S50. It exhibits a high lithium abundance, $A(\mathrm{Li})=2.14$, a value 12 times greater than solar. This fact is clearly evidenced when comparing the spectrum of this Li-rich star with that of a star in which $\mathrm{Li}$ is not detected (see Fig. 19). These stars, as S276 in NGC 6067 (Alonso-Santiago et al. 2017), are the coolest in the cluster and none of them exhibit a noticeable abundance of $\mathrm{Rb}$, which discards the remote possibility that they were AGBs (García-Hernández et al. 2006).

Reddy et al. (2016) did not compute Li abundances for any star but Holanda et al. (2019) give them for the five giants. Their values are displayed together with ours in Table 12 . When we compare both sets of abundances, we note that their values are significantly smaller than ours, specially for S50.

These Li-rich giants, a fraction $\sim 1 \%$ of all the giants analysed, have been found both in isolation (Brown et al. 1989) and within open clusters (Delgado Mena et al. 2016; AlonsoSantiago et al. 2017). Different scenarios have been proposed to explain this overabundace. The Cameron \& Fowler (1971) mechanism (CFM) can produce Li via the hot botton burning (Sackmann \& Boothroyd 1992) in intermediate-mass stars during the AGB phase as well as during the RGB phase in lowmass stars (Sackmann \& Boothroyd 1999), although it requires an extra mixing process. The engulfment of a sub-stellar companion (Siess \& Livio 1999; Aguilera-Gómez et al. 2016) or the enrichment of the interstellar medium after the explosion of an SN (Woosley \& Weaver 1995) could also make sense of the large amount of Li found.

As the cluster chemical composition (and S50 itself) is not above the Galactic average, the $\mathrm{SN}$ explosion scenario can be discarded. Given its very low abundance of $s$-process elements, such as Rb, and its position on the CMD, S50 should not be an AGB star, and so the CFM is also rejected (García-Hernández et al. 2006). After discarding all other possibilities, the engulfment of a planet or a brown dwarf by the star could be a plausible explanation, but our data cannot confirm or discard it. To shed light on this issue, we should determine the ${ }^{6} \mathrm{Li} /{ }^{7} \mathrm{Li}$ ratio, for which a spectrum with rather higher resolution is needed.

\section{Conclusions}

We offer an in-depth analysis of NGC 2345, the most complete to date. We provide the largest set of spectra, most of them for classification purposes, which combined with photometry and astrometry has allowed us to carry out a consistent analysis. For the first time, we used Strömgren photometry in addition to the 2MASS and Gaia DR2 archival data.

Our estimates for the cluster parameters such as the reddening (variable across the field, with a mean value of $E(B-V)=$ $0.66 \pm 0.13)$ and distance $(d=2.5 \pm 0.2 \mathrm{kpc})$ are compatible within the errors with those found in the literature. Also the age obtained in this work $(\tau=56 \pm 13 \mathrm{Ma})$ is consistent with the previous values, despite being slightly younger. This is as a consequence of taking into account the subsolar metallicity found by us instead of assuming a solar value as it had been done thus far. We computed a tidal radius, $r_{\mathrm{t}}=18.7 \pm 1.2$ arcmin, which is significantly larger than that obtained in previous works. Nevertheless, our result is consistent with the location of the most distant members, which is confirmed via radial velocity. Additionally, we suspect the existence of a large halo around the cluster.

We identify 145 likely blue members from which we derived an initial mass for the cluster around $5200 M_{\odot}$. This implies that, as in the case of NGC 3105, NGC 2345 is a moderately massive cluster that is comparable with NGC 7419, which can be used as a template for studying obscured RSGCs and for constraining evolutionary models of intermediate- and highmass stars. As in NGC 7419, we find a cluster with less than 

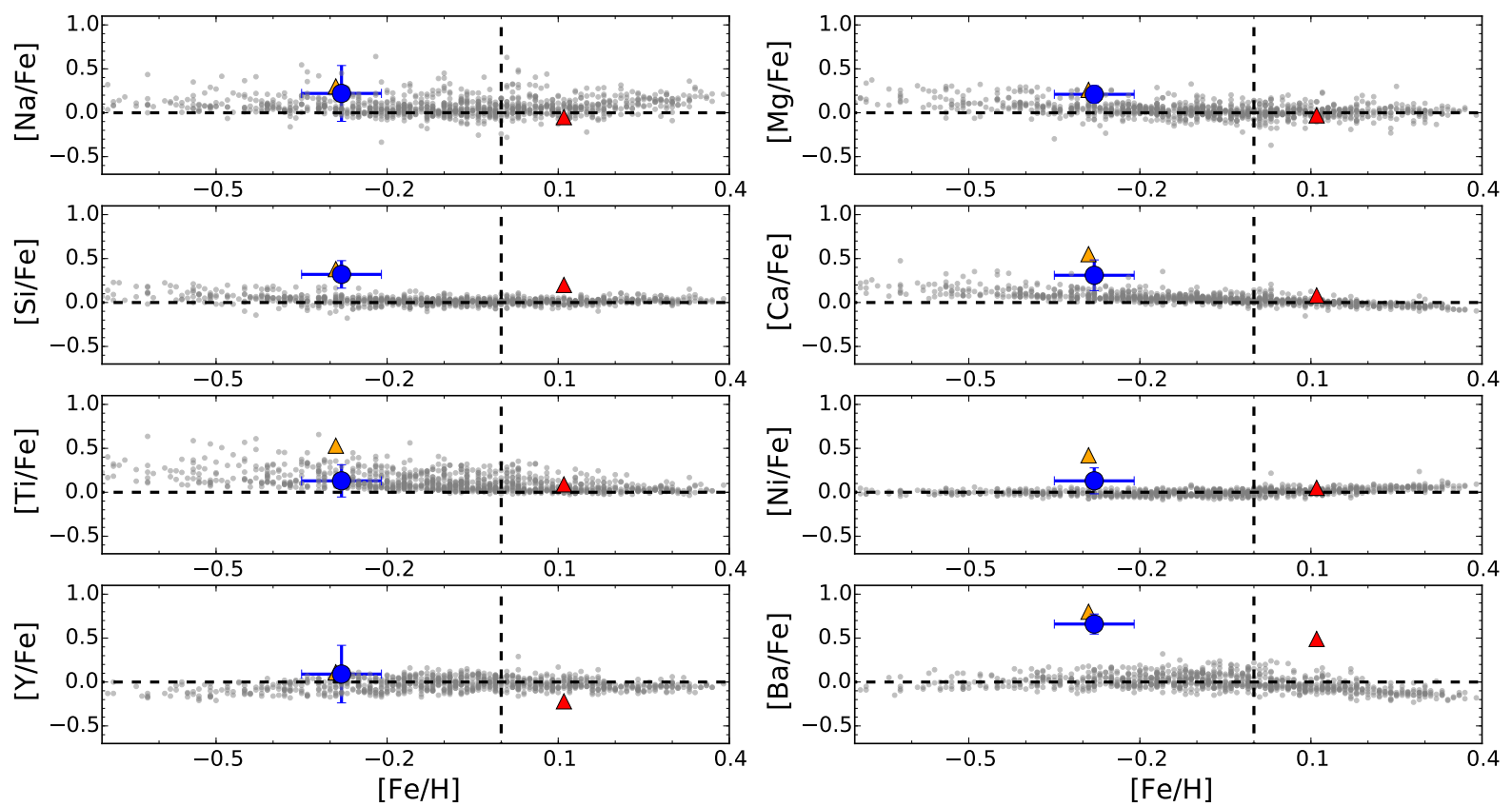

Fig. 18. Abundance ratios $[\mathrm{X} / \mathrm{Fe}]$ vs. $[\mathrm{Fe} / \mathrm{H}]$. The grey dots represent the Galactic trends for the thin disc (Adibekyan et al. 2012; Delgado Mena et al. 2017). NGC 6067 and NGC 3105 are drawn with triangles (orange and red, respectively) whereas NGC 2345 is the blue circle. Clusters are represented by their mean values. The dashed lines indicates the solar value.

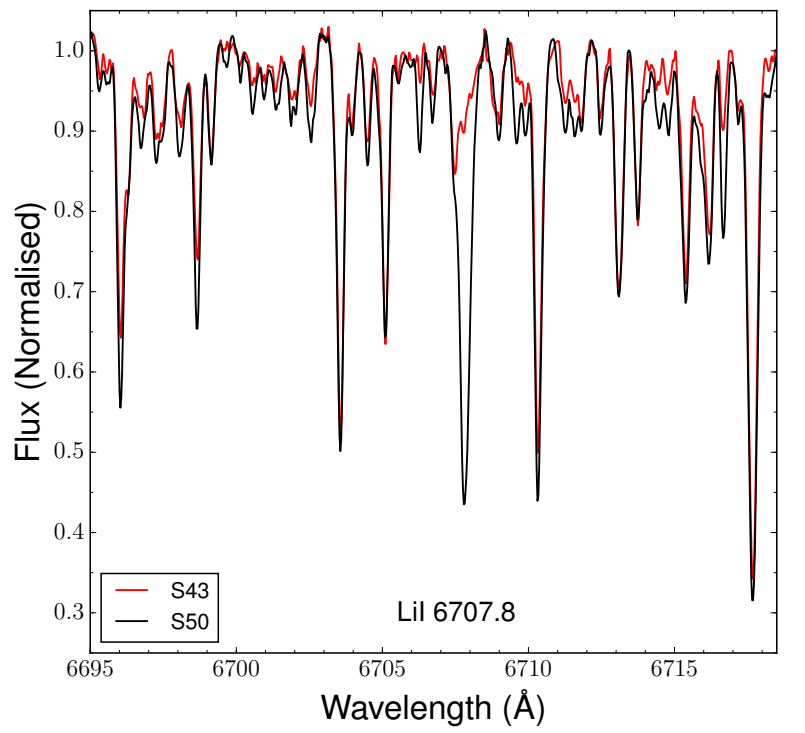

Fig. 19. Spectra around the $\mathrm{Li}$ line at $6707 \AA$. Li-rich giant, star 50 , is represented vs. star 43 , a Li-normal giant with similar stellar parameters.

$10000 M_{\odot}$ (in this case, significantly less), hosting six red (super)giants, with masses around $6.5 M_{\odot}$. One of them, S1000 (IRAS 07055-1302), has been identified for the first time in this work. Two other bright stars located in the halo, TYC 54021851-1 and TYC 5406-46-1, share the astrometric parameters of the cluster and, in addition, occupy a position on the CMD, which is compatible with that of the other red (super)giants. New spectroscopy is required to confirm their nature.

Similar to NGC 3105, NGC 2345 is located in the outer part of the Galaxy $\left(R_{\mathrm{GC}}=10.2 \pm 0.2 \mathrm{~km} \mathrm{~s}^{-1}\right)$ and shows a typical LMC metallicity $([\mathrm{Fe} / \mathrm{H}]=-0.28 \pm 0.07)$. This fact is probably related to the high $\mathrm{Be}$ fraction found $\geq 10.3 \%$ among all of the likely B-type members.
Table 12. Abundance of Li for five red giants in NGC 2345.

\begin{tabular}{lcc}
\hline \hline Star & Holanda19 ${ }^{(*)}$ & This work \\
\hline 14 & 0.95 & $1.67 \pm 0.09$ \\
34 & 0.11 & - \\
43 & 0.31 & - \\
50 & 0.81 & $2.14 \pm 0.03$ \\
60 & 0.19 & $<0.53$ \\
\hline
\end{tabular}

Notes. ${ }^{(*)}$ Typical uncertainties are \pm 0.1 dex.

We studied the chemical composition of NGC 2345 by computing the abundances of several chemical elements: Li, O, Na, and $\alpha$-elements (Mg, Si, Ca, and Ti); and the Fe-group (Ni) and $s$-elements ( $\mathrm{Rb}, \mathrm{Y}$, and $\mathrm{Ba})$. We find a homogeneous composition that is compatible with the Galactic trends observed in the thin disc. The coolest cluster member, S50, is found to be a Lirich $\operatorname{star}[A(\mathrm{Li})=2.11]$, as also happens in the case of NGC 6067, another young open cluster. Our results support the enhanced $s$ process model because of the overabundance of barium.

NGC 2345, as is the case with NGC 3105, and despite its unusually low metallicity, can be considered an excellent laboratory to improve theoretical models and an optimal template for the study of more obscured or unresolved clusters. Metallicity differences found along the Galactic disc affect the proper determination of the age of open clusters and, therefore, the mass of their evolved stars. Accordingly, metallicity must be considered as a key dimension in the study of the boundary between intermediate-mass and massive stars. Additional chemical studies of young open clusters will contribute to improving our understanding of the lower mass limit for SN progenitors.

Acknowledgements. This research is based on observations collected with the MPG/ESO 2.2-m Telescope operated at the La Silla Observatory (Chile) jointly by the Max Planck Institute for Astronomy and the European Organisation for Astronomical Research in the Southern Hemisphere under ESO programmes 
087.D-603(A) and 095.A-9020(A). The AAT observations and the second run taken with FEROS were supported by the OPTICON project (observing proposals 2010B/01 and 2015A/008, respectively), which is funded by the European Community's Seventh Framework Programme. The INT and WHT telescopes are operated on the island of La Palma by the Isaac Newton Group of Telescopes in the Spanish Observatorio del Roque de los Muchachos of the Instituto de Astrofísica de Canarias. The WFC photometry was obtained as part of 151-INT12/11B. The IDS spectroscopy was taken as part of 32-INT3/11A. WYFFOS spectra were obtained under proposal 94-WHT23/12B whereas ISIS spectroscopy was made in service mode as part of SW2011b13. This research is partially supported by the Spanish Government under grants AYA2015-68012 C2-2-P and PGC2018-093741-B-C21 (MICIU/AEI/FEDER, UE). This research has made use of the Simbad database, operated at CDS, Strasbourg (France). This publication also made use of data products from the Two Micron All Sky Survey, which is a joint project of the University of Massachusetts and the Infrared Processing and Analysis Center/California Institute of Technology, funded by the National Aeronautics and Space Administration and the National Science Foundation.

\section{References}

Adibekyan, V. Z., Sousa, S. G., Santos, N. C., et al. 2012, A\&A, 545, A32

Aguilera-Gómez, C., Chanamé, J., Pinsonneault, M. H., \& Carlberg, J. K. 2016, ApJ, 829, 127

Alonso-Santiago, J., Negueruela, I., Marco, A., et al. 2017, MNRAS, 469, 1330

Alonso-Santiago, J., Marco, A., Negueruela, I., et al. 2018, A\&A, 616, A124

Alonso-Santiago, J., Negueruela, I., Marco, A., \& Tabernero, H. M. 2019, in Highlights on Spanish Astrophysics X, Proceedings of the XIII Scientific Meeting of the Spanish Astronomical Society held on July 16-20, 2018, in Salamanca, Spain, eds. B. Montesinos, A. Asensio Ramos, F. Buitrago, et al. 261

Anderson, R. I., Eyer, L., \& Mowlavi, N. 2013, MNRAS, 434, 2238

Barklem, P. S., Piskunov, N., \& O'Mara, B. J. 2000, A\&AS, 142, 467

Bertran de Lis, S., Delgado Mena, E., Adibekyan, V. Z., Santos, N. C., \& Sousa, S. G. 2015, A\&A, 576, A89

Blanco-Cuaresma, S., Soubiran, C., Heiter, U., \& Jofré, P. 2014, A\&A, 569, A111

Bressan, A., Marigo, P., Girardi, L., et al. 2012, MNRAS, 427, 127

Brown, J. A., Sneden, C., Lambert, D. L., \& Dutchover, Jr., E. 1989, ApJS, 71, 293

Cameron, A. G. W., \& Fowler, W. A. 1971, ApJ, 164, 111

Cantat-Gaudin, T., Jordi, C., Vallenari, A, et al. 2018, A\&A, 618, A93

Carquillat, M. J., Jaschek, C., Jaschek, M., \& Ginestet, N. 1997, A\&AS, 123, 5

Carraro, G., Vázquez, R. A., Costa, E., Ahumada, J. A., \& Giorgi, E. E. 2015 AJ, 149,12

Castro, N., Urbaneja, M. A., Herrero, A., et al. 2012, A\&A, 542, A79

Charbonnel, C., \& Balachandran, S. C. 2000, A\&A, 359, 563

Crawford, D. L., \& Barnes, J. V. 1970, AJ, 75, 978

Crawford, D. L., Glaspey, J. W., \& Perry, C. L. 1970, AJ, 75, 822

Delgado Mena, E., Tsantaki, M., Sousa, S. G., et al. 2016, A\&A, 587, A66

Delgado Mena, E., Tsantaki, M., Adibekyan, V. Z., et al. 2017, A\&A, 606, A94

Doherty, C. L., Gil-Pons, P., Siess, L., Lattanzio, J. C., \& Lau, H. H. B. 2015 MNRAS, 446, 2599

D’Orazi, V., Magrini, L., Randich, S., et al. 2009, ApJ, 693, L31

D’Orazi, V., Lugaro, M., Campbell, S. W., et al. 2013, ApJ, 776, 59

Dutra-Ferreira, L., Pasquini, L., Smiljanic, R., Porto de Mello, G. F., \& Steffen, M. 2016, A\&A, 585, A75

Eggenberger, P., Meynet, G., \& Maeder, A. 2002, A\&A, 386, 576

Foreman-Mackey, D., Hogg, D. W., Lang, D., \& Goodman, J. 2013, PASP, 125, 306

Gaia Collaboration (Brown, A. G. A., et al.) 2018, A\&A, 616, A1

García-Hernández, D. A., García-Lario, P., Plez, B., et al. 2006, Science, 314, 1751

García-Hernández, D. A., García-Lario, P., Plez, B., et al. 2007, A\&A, 462, 711

Genovali, K., Lemasle, B., Bono, G., et al. 2013, A\&A, 554, A132

Genovali, K., Lemasle, B., Bono, G., et al. 2014, A\&A, 566, A37

Georgy, C., Ekström, S., Granada, A., et al. 2013, A\&A, 553, A24

González-Solares, E. A., Walton, N. A., Greimel, R., et al. 2008, MNRAS, 388, 89

González-Fernández, C., Dorda, R., Negueruela, I., \& Marco, A. 2015, A\&A, 578, A3

Granada, A., Georgy, C., Haemmerlé, L., Ekström, S., \& Meynet, G. 2016, in Bright Emissaries: Be Stars as Messengers of Star-Disk Physics, eds. T. A. A Sigut, \& C. E. Jones, ASP Conf. Ser., 506, 33
Gray, R. O., \& Corbally, C. J. 1994, AJ, 107, 742

Gray, R. O., \& Corbally, J. C. 2009, Stellar Spectral Classification (Princeton: Princeton University Press)

Grevesse, N., Asplund, M., \& Sauval, A. J. 2007, Space Sci. Rev., 130, 105

Gustafsson, B., Edvardsson, B., Eriksson, K., et al. 2008, A\&A, 486, 951

Heiter, U., \& Eriksson, K. 2006, A\&A, 452, 1039

Heiter, U., Soubiran, C., Netopil, M., \& Paunzen, E. 2014, A\&A, 561, A93

Holanda, N., Pereira, C. B., \& Drake, N. A. 2019, MNRAS, 482, 5275

Humphreys, R. M., \& McElroy, D. B. 1984, ApJ, 284, 565

Ibeling, D., \& Heger, A. 2013, ApJ, 765, L43

Iben, Jr., I. 1967a, ApJ, 147, 650

Iben, Jr., I. 1967b, ApJ, 147, 624

Ives, D. J., Tulloch, S., \& Churchill, J. 1996, in Solid State Sensor Arrays and CCD Cameras, eds. C. N. Anagnostopoulos, M. M. Blouke, \& M. P. Lesser, Proc. SPIE, 2654, 266

Jaschek, C., \& Jaschek, M. 1987, The Classification of Stars (Cambridge: Cambridge University Press)

Kang, W., \& Lee, S.-G. 2012, MNRAS, 425, 3162

Keller, S. C., Wood, P. R., \& Bessell, M. S. 1999, A\&AS, 134, 489

King, I. 1962, AJ, 67, 471

Kroupa, P. 2001, MNRAS, 322, 231

Kupka, F. G., Ryabchikova, T. A., Piskunov, N. E., Stempels, H. C., \& Weiss, W. W. 2000, Baltic Astron., 9, 590

Lefever, K. 2007, PhD Thesis, Institute of Astronomy, Katholieke Universiteit Leuven, Belgium

Maeder, A., \& Meynet, G. 2000, A\&A, 361, 159

Maíz Apellániz, J., \& Weiler, M. 2018, A\&A, 619, A180

Marco, A., \& Negueruela, I. 2013, A\&A, 552, A92

Martayan, C., Frémat, Y., Hubert, A.-M., et al. 2007, A\&A, 462, 683

Mathew, B., \& Subramaniam, A. 2011, Bull. Astron. Soc. India, 39, 517

Mathew, B., Subramaniam, A., \& Bhatt, B. C. 2008, MNRAS, 388, 1879

McSwain, M. V., \& Gies, D. R. 2005, in The Nature and Evolution of Disks Around Hot Stars, eds. R. Ignace, \& K. G. Gayley, ASP Conf. Ser., 337, 270

Mermilliod, J. C. 1982a, A\&A, 109, 48

Mermilliod, J.-C. 1982b, A\&A, 109, 37

Mermilliod, J. C., Mayor, M., \& Udry, S. 2008, A\&A, 485, 303

Mishenina, T., Korotin, S., Carraro, G., Kovtyukh, V. V., \& Yegorova, I. A. 2013, MNRAS, 433, 1436

Moffat, A. F. J. 1974, A\&AS, 16, 33

Negueruela, I., Alonso-Santiago, J., Tabernero, H. M., et al. 2017, Mem. Soc. Astron. It., 88, 368

Negueruela, I., Monguió, M., Marco, A., et al. 2018, MNRAS, 477, 2976

Netopil, M., Paunzen, E., Heiter, U., \& Soubiran, C. 2016, A\&A, 585, A150

Padmanabhan, N., Schlegel, D. J., Finkbeiner, D. P., et al. 2008, ApJ, 674, 1217

Perry, C. L., Olsen, E. H., \& Crawford, D. L. 1987, PASP, 99, 1184

Pigulski, A., Kopacki, G., \& Kołaczkowski, Z. 2001, A\&A, 376, 144

Piskunov, N. E., Kupka, F., Ryabchikova, T. A., Weiss, W. W., \& Jeffery, C. S. 1995, A\&AS, 112, 525

Piskunov, A. E., Schilbach, E., Kharchenko, N. V., Röser, S., \& Scholz, R.-D. 2008, A\&A, 477, 165

Plez, B. 2012, Astrophysics Source Code Library [record ascl:1205 . 004]

Podsiadlowski, P., Langer, N., Poelarends, A. J. T., et al. 2004, ApJ, 612, 1044

Poelarends, A. J. T., Herwig, F., Langer, N., \& Heger, A. 2008, ApJ, 675, 614

Porter, J. M., \& Rivinius, T. 2003, PASP, 115, 1153

Puls, J., Urbaneja, M. A., Venero, R., et al. 2005, A\&A, 435, 669

Pumo, M. L., Turatto, M., Botticella, M. T., et al. 2009, ApJ, 705, L138

Reddy, A. B. S., Lambert, D. L., \& Giridhar, S. 2016, MNRAS, 463, 4366

Sackmann, I.-J., \& Boothroyd, A. I. 1992, ApJ, 392, L71

Sackmann, I.-J., \& Boothroyd, A. I. 1999, ApJ, 510, 217

Santolaya-Rey, A. E., Puls, J., \& Herrero, A. 1997, A\&A, 323, 488

Siess, L., \& Livio, M. 1999, MNRAS, 308, 1133

Simón-Díaz, S., Castro, N., Herrero, A., et al. 2011, J. Phys. Conf. Ser., 328, 012021

Simón-Díaz, S., \& Herrero, A. 2014, A\&A, 562, A135

Skrutskie, M. F., Cutri, R. M., Stiening, R., et al. 2006, AJ, 131, 1163

Soderblom, D. R., Jones, B. F., Balachandran, S., et al. 1993, AJ, 106, 1059

Stephenson, C. B., \& Sanduleak, N. 1971, Publications of the Warner \& Swasey Observatory (Cleveland: Case Western Reserve University), 1

Stephenson, C. B., \& Sanduleak, N. 1977, ApJS, 33, 459

Tabernero, H. M., Dorda, R., Negueruela, I., \& González-Fernández, C. 2018 , MNRAS, 476, 3106

Walborn, N. R., \& Fitzpatrick, E. L. 1990, PASP, 102, 379

Woosley, S. E., \& Weaver, T. A. 1995, ApJS, 101, 181 


\section{Appendix A: Additional tables}

Table A.1. Strömgren photometry obtained in field of NGC 2345 in this work.

\begin{tabular}{|c|c|c|c|c|c|c|c|c|c|c|c|}
\hline ID & RA (J2000) & $\operatorname{Dec}(\mathrm{J} 2000)$ & $V$ & $\sigma_{V}$ & $(b-y)$ & $\sigma_{(b-y)}$ & $m_{1}$ & $\sigma_{m_{1}}$ & $c_{1}$ & $\sigma_{c_{1}}$ & $N$ \\
\hline 1 & 106.675384521 & -13.389435768 & 16.908 & 0.020 & 0.253 & 0.027 & 1.218 & 0.045 & -0.800 & 0.028 & 1 \\
\hline 2 & 106.676712036 & -13.385466576 & 16.514 & 0.025 & 0.556 & 0.025 & 1.055 & 0.043 & -0.669 & 0.030 & 1 \\
\hline 3 & 106.677474976 & -13.152485847 & 16.785 & 0.017 & 0.003 & 0.025 & 1.042 & 0.039 & -0.254 & 0.021 & 1 \\
\hline 4 & 106.677742004 & -13.337368011 & 16.028 & 0.022 & 0.508 & 0.022 & 0.976 & 0.039 & -0.224 & 0.025 & 1 \\
\hline 5 & 106.678443909 & -13.416952133 & 15.156 & 0.050 & 0.567 & 0.059 & 0.593 & 0.065 & -0.030 & 0.070 & 2 \\
\hline 6 & 106.679504395 & -13.219663620 & 16.263 & 0.059 & 0.779 & 0.196 & -0.101 & 0.224 & 0.425 & 0.242 & 2 \\
\hline 7 & 106.679794312 & -13.376412392 & 14.627 & 0.036 & 0.986 & 0.032 & -0.074 & 0.045 & 0.519 & 0.037 & 3 \\
\hline 8 & 106.679885864 & -13.230211258 & 16.126 & 0.080 & 1.071 & 0.161 & 0.247 & 0.180 & 0.037 & 0.184 & 2 \\
\hline 9 & 106.680000305 & -13.412867546 & 17.148 & 0.067 & 0.443 & 0.204 & 0.622 & 0.210 & -0.136 & 0.210 & 3 \\
\hline 10 & 106.680206299 & -13.457053185 & 16.875 & 0.044 & 0.495 & 0.105 & 0.050 & 0.103 & 1.727 & 0.100 & 2 \\
\hline
\end{tabular}

Notes. Only ten rows are listed here for guidance regarding its form and content. Full table is available at the CDS.

Table A.2. For every star with spectroscopy the equatorial coordinates, spectral type, radial velocity (from the AA $\Omega$ spectrum), spectrographs used, and cluster membership are displayed.

\begin{tabular}{|c|c|c|c|c|c|c|c|c|c|c|c|}
\hline Star & ID & RA (J2000) & Dec (J2000) & $\mathrm{Sp} \mathrm{T}$ & $V_{\mathrm{rad}}\left(\mathrm{km} \mathrm{s}^{-1}\right)$ & $\mathrm{AA} \Omega$ & WYFFOS & FEROS & ISIS & IDS & Member \\
\hline 1 & 1239 & 107.060083333 & -13.268638889 & A2 Ib & 66 & - & & - & & - & $\mathrm{y}$ \\
\hline 2 & 1148 & 107.043750000 & -13.260222222 & B5 IIIe & 74 & - & - & & & & $\mathrm{y}$ \\
\hline 4 & 970 & 107.007291667 & -13.228638889 & B4 III & 62 & - & - & & - & - & $\mathrm{y}$ \\
\hline 5 & 1074 & 107.031583333 & -13.222833333 & B7 Ve & 30 & - & & & & & $\mathrm{y}$ \\
\hline 6 & 1191 & 107.051375000 & -13.229638889 & B6 V & 56 & - & - & & & & $\mathrm{y}$ \\
\hline 7 & 1265 & 107.064458333 & -13.229694444 & A3 Ib & 56 & - & & - & & - & $\mathrm{y}$ \\
\hline 14 & 1320 & 107.072791667 & -13.191805556 & K3 Ib & 59 & - & & - & & & $\mathrm{y}$ \\
\hline 20 & 1195 & 107.052125000 & -13.177083333 & B5 Ve & 61 & - & & & & & $\mathrm{y}$ \\
\hline 22 & 1047 & 107.025250000 & -13.186194444 & B4 III & 57 & - & & & - & - & $\mathrm{y}$ \\
\hline 23 & 1063 & 107.029000000 & -13.161388889 & B3 V & 57 & - & - & & & & $\mathrm{y}$ \\
\hline 24 & 1171 & 107.048458333 & -13.157500000 & B3 IIIe & 56 & - & - & & & & $\mathrm{y}$ \\
\hline 25 & 1234 & 107.059708333 & -13.169861111 & B3 V & 75 & - & & & & & $\mathrm{y}$ \\
\hline 28 & 1031 & 107.069958333 & -13.165750000 & B3 IV & 58 & - & - & - & - & - & $\mathrm{y}$ \\
\hline 30 & 1374 & 107.080458333 & -13.116944444 & B7 IIIe & 54 & - & - & & & & $\mathrm{y}$ \\
\hline 32 & 1385 & 107.081375000 & -13.161805556 & $\mathrm{~B} 5 \mathrm{Ve}$ & 55 & - & & & & & $\mathrm{y}$ \\
\hline 34 & 1451 & 107.091083333 & -13.173111111 & G6 II + B & 64 & - & & - & & & $\mathrm{y}$ \\
\hline 35 & 1472 & 107.095250000 & -13.171888889 & B4 IIIe & 58 & - & - & & - & - & $\mathrm{y}$ \\
\hline 36 & 1767 & 107.148500000 & -13.166083333 & A2 V & -6 & - & - & & & & $\mathrm{n}$ \\
\hline 37 & 1680 & 107.133125000 & -13.169944444 & B3 IV & 61 & - & & & & & $\mathrm{y}$ \\
\hline 39 & 1723 & 107.140375000 & -13.182750000 & B5 IV & 56 & - & - & & & & $\mathrm{y}$ \\
\hline 43 & 1553 & 107.109687500 & -13.187344444 & K0 Ib-II & 58 & & & - & & & $\mathrm{y}$ \\
\hline 44 & 1526 & 107.106291667 & -13.200666667 & B5 Ve & 52 & - & - & & & & $\mathrm{y}$ \\
\hline 47 & 1623 & 107.122000000 & -13.212591667 & B4 V & 56 & & & & - & - & $\mathrm{n}$ \\
\hline 50 & 1568 & 107.112541667 & -13.209166667 & $\mathrm{~K} 4 \mathrm{Ib}$ & 59 & - & & - & & & $\mathrm{y}$ \\
\hline 51 & 1558 & 107.109875000 & -13.210166667 & B3 IV & 58 & - & - & & - & - & $\mathrm{y}$ \\
\hline 54 & 1506 & 107.103041667 & -13.229388889 & B5 V & 43 & - & - & & & & $\mathrm{y}$ \\
\hline 56 & 1544 & 107.108666667 & -13.240111111 & B6 V & 49 & - & & & & & $\mathrm{y}$ \\
\hline 59 & 1595 & 107.116791667 & -13.259805556 & B5 Ve & 54 & - & & & & & $\mathrm{y}$ \\
\hline 60 & 1646 & 107.126541667 & -13.231250000 & K2 Ib-II & 58 & - & & - & & & $\mathrm{y}$ \\
\hline 61 & 1640 & 107.124791667 & -13.220888889 & B7 Ve & 50 & - & - & & & & $\mathrm{y}$ \\
\hline 63 & & 107.294583333 & -13.181111111 & B5 Ve & 44 & - & - & & & & $\mathrm{y}$ \\
\hline 64 & 899 & 106.988375000 & -13.058833333 & B3 IV & 59 & - & - & & & & $\mathrm{y}$ \\
\hline 1000 & 873 & 106.982500000 & -13.128416667 & $\mathrm{~K} 4-5 \mathrm{Ib}$ & 59 & $\bullet$ & & - & & & $\mathrm{y}$ \\
\hline 1002 & & 107.297083333 & -13.434694444 & M6 II & & - & & - & & & $\mathrm{n}$ \\
\hline 1003 & 811 & 106.964625000 & -13.076305556 & B5 Ve & 48 & - & - & & & & $\mathrm{y}$ \\
\hline 1004 & & 107.575791667 & -12.703333333 & B3 V & 32 & - & & & & & $\mathrm{n}$ \\
\hline 1005 & & 107.487541667 & -13.616055556 & G4 II & & - & & & & & $\mathrm{n}$ \\
\hline
\end{tabular}


Table A.2. continued.

\begin{tabular}{|c|c|c|c|c|c|c|c|c|c|c|c|}
\hline Star & ID & RA (J2000) & $\operatorname{Dec}(\mathrm{J} 2000)$ & $\mathrm{Sp} \mathrm{T}$ & $V_{\mathrm{rad}}\left(\mathrm{km} \mathrm{s}^{-1}\right)$ & $\mathrm{AA} \Omega$ & WYFFOS & FEROS & ISIS & IDS & Member \\
\hline 1009 & & 106.442000000 & -13.160305556 & B0.5e & & $\bullet$ & $\bullet$ & & & & $\mathrm{n}$ \\
\hline 1010 & & 107.355875000 & -12.541916667 & $\mathrm{BN} 2 \mathrm{Ib}$ & 49 & $\bullet$ & & & & & $\mathrm{n}$ \\
\hline 1011 & 1387 & 107.081083333 & -12.980361111 & F V: & 40 & $\bullet$ & $\bullet$ & & & & $\mathrm{n}$ \\
\hline 1012 & 902 & 106.990291667 & -13.161388889 & B3 V & 47 & $\bullet$ & $\bullet$ & & & & $\mathrm{y}$ \\
\hline 1020 & 586 & 106.901708333 & -13.264388889 & B8 III & 59 & $\bullet$ & $\bullet$ & & & & $\mathrm{n}$ \\
\hline 1021 & 427 & 106.855083333 & -13.295472222 & late A III & 39 & $\bullet$ & $\bullet$ & & & & $\mathrm{n}$ \\
\hline 1022 & 981 & 107.010583333 & -13.436277778 & B3 V & 31 & $\bullet$ & $\bullet$ & & & & $\mathrm{n}$ \\
\hline 1023 & & 107.355958333 & -12.926277778 & B5 V & 12 & $\bullet$ & $\bullet$ & & & & $\mathrm{n}$ \\
\hline 1024 & & 107.288833333 & -12.962666667 & late A III & 36 & $\bullet$ & $\bullet$ & & & & $\mathrm{n}$ \\
\hline 1025 & & 107.261416667 & -12.961500000 & A3 V & 14 & $\bullet$ & $\bullet$ & & & & $\mathrm{n}$ \\
\hline 1026 & & 107.308041667 & -12.942138889 & early A & 21 & $\bullet$ & & & & & $\mathrm{n}$ \\
\hline 1027 & & 107.327375000 & -13.042611111 & A5 V & 66 & $\bullet$ & $\bullet$ & & & & $\mathrm{n}$ \\
\hline 1028 & & 107.385375000 & -13.076972222 & A0 III & 22 & $\bullet$ & $\bullet$ & & & & $\mathrm{n}$ \\
\hline 1029 & & 107.375083333 & -13.127833333 & A7 II-III & 23 & $\bullet$ & $\bullet$ & & & & $\mathrm{n}$ \\
\hline 1030 & & 106.788125000 & -12.978861111 & A2 III & 15 & $\bullet$ & $\bullet$ & & & & $\mathrm{n}$ \\
\hline 1031 & & 106.809375000 & -12.979916667 & B9.5 II-III & 65 & $\bullet$ & $\bullet$ & & & & $\mathrm{n}$ \\
\hline 1032 & 306 & 106.803875000 & -13.216527778 & A1 V & 30 & $\bullet$ & $\bullet$ & & & & $\mathrm{n}$ \\
\hline 1034 & 1704 & 107.136500000 & -12.986277778 & early F & 0 & $\bullet$ & $\bullet$ & & & & $\mathrm{n}$ \\
\hline 1035 & 1539 & 107.107791667 & -13.004750000 & A0 V & 13 & $\bullet$ & $\bullet$ & & & & $\mathrm{n}$ \\
\hline 1050 & & 107.602041667 & -13.564472222 & A: & 36 & $\bullet$ & & & & & $\mathrm{n}$ \\
\hline 1051 & & 107.149833333 & -13.989472222 & B5 III shell & 78 & $\bullet$ & & & & & $\mathrm{n}$ \\
\hline 1125 & & 106.382750000 & -13.401666667 & B5 III & 37 & $\bullet$ & & & & & $\mathrm{n}$ \\
\hline 1126 & & 106.368125000 & -13.353416667 & B8 III & 15 & $\bullet$ & & & & & $\mathrm{n}$ \\
\hline LS 152 & & 106.406416667 & -13.523333333 & B2 IV & 64 & $\bullet$ & & & & & $\mathrm{n}$ \\
\hline LS 153 & & 106.404791667 & -13.775888889 & B0.7 III & 91 & $\bullet$ & & & & & $\mathrm{n}$ \\
\hline LS 164 & & 106.556541667 & -13.185861111 & $08 \mathrm{~V}((\mathrm{f})) \mathrm{z}$ & 70 & $\bullet$ & $\bullet$ & & & & $\mathrm{n}$ \\
\hline LS 165 & & 106.605083333 & -13.800444444 & B0.7 III & & $\bullet$ & & & & & $\mathrm{n}$ \\
\hline LS 170 & 86 & 106.715083333 & -13.287694444 & B9 Ib & 99 & $\bullet$ & $\bullet$ & & & & $\mathrm{n}$ \\
\hline LS 171 & & 106.734958333 & -12.774555556 & $\mathrm{~B} 1.5 \mathrm{Ve}$ & 71 & $\bullet$ & $\bullet$ & & & & $\mathrm{n}$ \\
\hline LS 174 & & 106.813041667 & -13.659750000 & $\mathrm{~B} 2 \mathrm{Ve}$ & 57 & $\bullet$ & $\bullet$ & & & & $\mathrm{y} ?$ \\
\hline LS 176 & & 106.955375000 & -12.787944444 & B3 IIIe & 65 & $\bullet$ & $\bullet$ & & & & $\mathrm{y} ?$ \\
\hline LS 180 & & 107.029333333 & -12.805333333 & B5 V & 62 & $\bullet$ & $\bullet$ & & & & $\mathrm{n}$ \\
\hline LS 193 & 2093 & 107.242875000 & -13.432277778 & B3 IV & 47 & $\bullet$ & $\bullet$ & $\bullet$ & & & $\mathrm{y}$ \\
\hline LS 205 & & 107.489291667 & -13.502833333 & B3 III & 72 & $\bullet$ & & & & & y? \\
\hline LS 215 & & 107.646958333 & -13.053305556 & & 42 & $\bullet$ & & & & & $\mathrm{n}$ \\
\hline LS 221 & & 107.748666667 & -13.526222222 & & & $\bullet$ & & & & & $\mathrm{n}$ \\
\hline LS 224 & & 107.809166667 & -13.510833333 & & 33 & $\bullet$ & & & & & $\mathrm{n}$ \\
\hline LS 229 & & 107.885583333 & -12.844277778 & B2.5 III & 72 & $\bullet$ & & & & & $\mathrm{y} ?$ \\
\hline HD 54743 & & 107.373333333 & -13.176000000 & G5 III & 8 & - & & & & & $\mathrm{n}$ \\
\hline
\end{tabular}

Table A.3. Gaia DR2 astrometric data for all stars in field of NGC 2345 for which we have spectra and other objects discussed in text.

\begin{tabular}{lccc}
\hline \hline Star & $\varpi(\mathrm{mas})$ & $\mu_{\alpha *}\left(\mathrm{mas} \mathrm{a}^{-1}\right)$ & $\mu_{\delta}\left(\mathrm{mas} \mathrm{a}^{-1}\right)$ \\
\hline \multicolumn{4}{c}{ Members } \\
\hline 1 & $0.3615 \pm 0.0342$ & $-1.537 \pm 0.054$ & $1.472 \pm 0.048$ \\
2 & $0.3507 \pm 0.0330$ & $-1.413 \pm 0.050$ & $1.351 \pm 0.045$ \\
4 & $0.3904 \pm 0.0343$ & $-1.360 \pm 0.052$ & $1.415 \pm 0.046$ \\
5 & $0.3464 \pm 0.0214$ & $-1.392 \pm 0.031$ & $1.409 \pm 0.028$ \\
6 & $0.3154 \pm 0.0214$ & $-1.213 \pm 0.032$ & $1.313 \pm 0.029$ \\
7 & $0.3906 \pm 0.0361$ & $-1.446 \pm 0.052$ & $1.248 \pm 0.046$ \\
14 & $0.4448 \pm 0.0592$ & $-1.692 \pm 0.087$ & $1.536 \pm 0.079$ \\
20 & $0.3261 \pm 0.0232$ & $-1.321 \pm 0.032$ & $1.287 \pm 0.030$ \\
22 & $0.3687 \pm 0.0339$ & $-1.355 \pm 0.052$ & $1.330 \pm 0.047$ \\
23 & $0.3615 \pm 0.0216$ & $-1.401 \pm 0.030$ & $1.325 \pm 0.027$ \\
24 & $0.3671 \pm 0.0226$ & $-1.390 \pm 0.032$ & $1.310 \pm 0.029$ \\
25 & $0.3723 \pm 0.0234$ & $-1.242 \pm 0.032$ & $1.236 \pm 0.029$ \\
27 & $0.3170 \pm 0.0239$ & $-1.378 \pm 0.032$ & $1.322 \pm 0.029$ \\
\hline
\end{tabular}


Table A.3. continued.

\begin{tabular}{|c|c|c|c|}
\hline Star & $\varpi$ (mas) & $\mu_{\alpha *}\left(\mathrm{mas} \mathrm{a}^{-1}\right)$ & $\mu_{\delta}\left(\mathrm{mas} \mathrm{a}^{-1}\right)$ \\
\hline 28 & $0.3653 \pm 0.0363$ & $-1.336 \pm 0.050$ & $1.270 \pm 0.045$ \\
\hline 30 & $0.3675 \pm 0.0246$ & $-1.341 \pm 0.034$ & $1.309 \pm 0.032$ \\
\hline 32 & $0.3378 \pm 0.0234$ & $-1.353 \pm 0.032$ & $1.324 \pm 0.029$ \\
\hline 34 & $0.3670 \pm 0.0390$ & $-1.201 \pm 0.055$ & $1.238 \pm 0.050$ \\
\hline 35 & $0.2823 \pm 0.0372$ & $-1.464 \pm 0.052$ & $1.387 \pm 0.048$ \\
\hline 37 & $0.3930 \pm 0.0330$ & $-1.284 \pm 0.048$ & $1.357 \pm 0.044$ \\
\hline 39 & $0.3335 \pm 0.0322$ & $-1.327 \pm 0.048$ & $1.342 \pm 0.043$ \\
\hline 43 & $0.3926 \pm 0.0351$ & $-1.241 \pm 0.050$ & $1.238 \pm 0.045$ \\
\hline 44 & $0.3465 \pm 0.0241$ & $-1.280 \pm 0.032$ & $1.295 \pm 0.028$ \\
\hline 50 & $0.3386 \pm 0.0434$ & $-1.273 \pm 0.063$ & $1.179 \pm 0.058$ \\
\hline 51 & $0.3321 \pm 0.0350$ & $-1.413 \pm 0.051$ & $1.505 \pm 0.053$ \\
\hline 54 & $0.3239 \pm 0.0404$ & $-1.293 \pm 0.058$ & $1.265 \pm 0.049$ \\
\hline 56 & $0.3234 \pm 0.0241$ & $-1.425 \pm 0.035$ & $1.230 \pm 0.032$ \\
\hline 59 & $0.3431 \pm 0.0281$ & $-1.360 \pm 0.042$ & $1.489 \pm 0.037$ \\
\hline 60 & $0.3457 \pm 0.0380$ & $-1.403 \pm 0.051$ & $1.431 \pm 0.046$ \\
\hline 61 & $0.3407 \pm 0.0320$ & $-1.336 \pm 0.042$ & $1.384 \pm 0.037$ \\
\hline 63 & $0.3558 \pm 0.0335$ & $-1.295 \pm 0.051$ & $1.336 \pm 0.046$ \\
\hline 64 & $0.3564 \pm 0.0427$ & $-1.382 \pm 0.060$ & $1.345 \pm 0.052$ \\
\hline 1000 & $0.3932 \pm 0.0539$ & $-1.712 \pm 0.078$ & $0.950 \pm 0.066$ \\
\hline 1003 & $0.3517 \pm 0.0376$ & $-1.437 \pm 0.056$ & $1.328 \pm 0.050$ \\
\hline 1012 & $0.3796 \pm 0.0273$ & $-1.337 \pm 0.049$ & $1.269 \pm 0.039$ \\
\hline LS 193 & $0.3786 \pm 0.0386$ & $-1.231 \pm 0.059$ & $1.356 \pm 0.050$ \\
\hline $\mathrm{X} 1$ & $0.3481 \pm 0.0221$ & $-1.343 \pm 0.033$ & $1.359 \pm 0.030$ \\
\hline $\mathrm{X} 2$ & $0.3531 \pm 0.0236$ & $-1.276 \pm 0.032$ & $1.287 \pm 0.028$ \\
\hline \multicolumn{4}{|c|}{ Halo members } \\
\hline LS 174 & $0.3070 \pm 0.0415$ & $-1.253 \pm 0.055$ & $1.578 \pm 0.056$ \\
\hline LS 176 & $0.2792 \pm 0.0330$ & $-1.228 \pm 0.062$ & $1.278 \pm 0.058$ \\
\hline LS 205 & $0.2749 \pm 0.0328$ & $-1.129 \pm 0.045$ & $1.220 \pm 0.041$ \\
\hline LS 229 & $0.2906 \pm 0.0564$ & $-1.182 \pm 0.083$ & $1.037 \pm 0.077$ \\
\hline TYC-5402-1851-1 & $0.3168 \pm 0.0479$ & $-1.067 \pm 0.066$ & $1.290 \pm 0.059$ \\
\hline TYC-5406-46-1 & $0.2941 \pm 0.0406$ & $-1.424 \pm 0.084$ & $1.254 \pm 0.076$ \\
\hline \multicolumn{4}{|c|}{ Non members } \\
\hline 36 & $1.0756 \pm 0.0350$ & $+0.525 \pm 0.051$ & $3.257 \pm 0.046$ \\
\hline 47 & $0.0802 \pm 0.1666$ & $+2.770 \pm 0.259$ & $-3.205 \pm 0.222$ \\
\hline 1002 & $0.4178 \pm 0.1514$ & $-2.556 \pm 0.228$ & $1.936 \pm 0.196$ \\
\hline 1004 & $0.6156 \pm 0.0601$ & $-1.781 \pm 0.094$ & $-1.044 \pm 0.090$ \\
\hline 1005 & $2.1214 \pm 0.0507$ & $-3.566 \pm 0.070$ & $-7.884 \pm 0.061$ \\
\hline 1009 & $0.0537 \pm 0.0383$ & $-0.152 \pm 0.064$ & $0.780 \pm 0.064$ \\
\hline 1010 & $0.3709 \pm 0.0475$ & $-1.240 \pm 0.070$ & $1.481 \pm 0.073$ \\
\hline 1011 & $1.0805 \pm 0.0372$ & $-1.817 \pm 0.057$ & $4.019 \pm 0.052$ \\
\hline 1020 & $0.3252 \pm 0.0257$ & $-0.620 \pm 0.038$ & $0.364 \pm 0.036$ \\
\hline 1021 & $0.9576 \pm 0.0426$ & $-4.399 \pm 0.074$ & $0.117 \pm 0.069$ \\
\hline 1022 & $0.5039 \pm 0.0382$ & $-2.033 \pm 0.062$ & $1.170 \pm 0.056$ \\
\hline 1023 & $0.4986 \pm 0.0415$ & $-1.908 \pm 0.061$ & $1.130 \pm 0.055$ \\
\hline 1024 & $0.8040 \pm 0.0329$ & $-3.270 \pm 0.053$ & $2.525 \pm 0.046$ \\
\hline 1025 & $0.7865 \pm 0.0227$ & $-3.007 \pm 0.035$ & $0.544 \pm 0.033$ \\
\hline 1026 & $0.3746 \pm 0.0224$ & $0.019 \pm 0.036$ & $-0.508 \pm 0.031$ \\
\hline 1027 & $1.1490 \pm 0.0329$ & $-3.297 \pm 0.052$ & $3.115 \pm 0.046$ \\
\hline 1028 & $0.9284 \pm 0.0335$ & $-3.725 \pm 0.051$ & $0.382 \pm 0.045$ \\
\hline 1029 & $1.2657 \pm 0.0411$ & $-4.539 \pm 0.072$ & $2.401 \pm 0.067$ \\
\hline 1030 & $-1.9468 \pm 0.5428$ & $1.207 \pm 1.042$ & $-4.808 \pm 0.986$ \\
\hline 1031 & $0.4076 \pm 0.0222$ & $-0.548 \pm 0.035$ & $-0.121 \pm 0.037$ \\
\hline 1032 & $0.3987 \pm 0.0395$ & $-1.670 \pm 0.054$ & $0.007 \pm 0.052$ \\
\hline 1034 & $1.1756 \pm 0.0398$ & $0.869 \pm 0.061$ & $1.108 \pm 0.054$ \\
\hline 1035 & $0.9189 \pm 0.0385$ & $-3.373 \pm 0.060$ & $0.694 \pm 0.052$ \\
\hline 1050 & $0.7041 \pm 0.0402$ & $-0.970 \pm 0.061$ & $-0.118 \pm 0.054$ \\
\hline 1051 & $4.1798 \pm 0.0641$ & $-2.717 \pm 0.086$ & $-4.901 \pm 0.076$ \\
\hline 1125 & $0.3853 \pm 0.0511$ & $-2.040 \pm 0.077$ & $1.022 \pm 0.074$ \\
\hline
\end{tabular}


Table A.3. continued.

\begin{tabular}{lccc}
\hline \hline Star & $\varpi$ (mas) & $\mu_{\alpha *}\left(\mathrm{mas} \mathrm{a}^{-1}\right)$ & $\mu_{\delta}\left(\mathrm{mas} \mathrm{a}^{-1}\right)$ \\
\hline 1126 & $0.6767 \pm 0.0507$ & $-0.663 \pm 0.069$ & $0.131 \pm 0.067$ \\
LS 152 & $0.3871 \pm 0.0394$ & $-1.068 \pm 0.067$ & $0.269 \pm 0.065$ \\
LS 153 & $0.0337 \pm 0.0354$ & $-0.321 \pm 0.058$ & $0.804 \pm 0.053$ \\
LS 164 & $-0.0236 \pm 0.0612$ & $-0.396 \pm 0.076$ & $0.852 \pm 0.085$ \\
LS 165 & $0.0732 \pm 0.0415$ & $-0.421 \pm 0.064$ & $0.958 \pm 0.059$ \\
LS 170 & $0.0948 \pm 0.1013$ & $0.029 \pm 0.139$ & $0.881 \pm 0.153$ \\
LS 171 & $0.0590 \pm 0.0349$ & $-0.250 \pm 0.062$ & $1.553 \pm 0.057$ \\
LS 180 & $0.3845 \pm 0.0447$ & $-2.089 \pm 0.069$ & $2.350 \pm 0.065$ \\
LS 215 & $0.4011 \pm 0.0385$ & $-0.861 \pm 0.056$ & $1.268 \pm 0.059$ \\
LS 221 & $0.1266 \pm 0.0335$ & $-1.053 \pm 0.050$ & $0.998 \pm 0.046$ \\
LS 224 & $0.2552 \pm 0.0289$ & $-0.902 \pm 0.044$ & $1.112 \pm 0.040$ \\
HD 54743 & $1.5844 \pm 0.0381$ & $-9.664 \pm 0.056$ & $-0.122 \pm 0.050$ \\
TV CMa & $0.3137 \pm 0.0335$ & $-0.743 \pm 0.050$ & $0.104 \pm 0.045$ \\
ASAS J070911-1217.2 & $0.3140 \pm 0.0564$ & $-1.045 \pm 0.082$ & $1.276 \pm 0.091$ \\
\hline
\end{tabular}

Table A.4. Photometric and astrometric data for likely blue members of NGC 2345.

\begin{tabular}{ccccccccccc}
\hline \hline ID & $E(B-V)$ & $V$ & $(b-y)$ & $J$ & $\left(J-K_{S}\right)$ & $G$ & $\left(G_{\mathrm{BP}}-G_{\mathrm{RP}}\right)$ & $\varpi(\mathrm{mas})$ & $\mu_{\alpha *}\left(\mathrm{mas} \mathrm{a}^{-1}\right)$ & $\mu_{\delta}\left(\mathrm{mas} \mathrm{a}^{-1}\right)$ \\
\hline 131 & 0.757 & 15.973 & 0.549 & 14.442 & 0.434 & 15.789 & 0.980 & 0.422 & -1.419 \\
137 & 0.576 & 14.363 & 0.380 & 13.167 & 0.296 & 14.222 & 0.705 & 0.345 & -1.336 \\
140 & 0.849 & 16.038 & 0.604 & 14.317 & 0.461 & 15.762 & 1.078 & 0.389 & -1.113 & 1.424 \\
169 & 1.130 & 15.273 & 0.784 & 13.017 & 0.435 & 14.916 & 1.426 & 0.318 & -1.188 & 1.235 \\
242 & 0.480 & 14.311 & 0.326 & 13.346 & 0.139 & 14.234 & 0.591 & 0.290 & -1.411 & 1.436 \\
243 & 0.859 & 16.133 & 0.613 & 14.345 & 0.535 & 15.939 & 1.172 & 0.420 & -1.387 & 1.310 \\
248 & 0.769 & 14.916 & 0.547 & 13.494 & 0.344 & 14.785 & 0.957 & 0.371 & -1.697 & 1.203 \\
307 & 0.757 & 15.624 & 0.542 & 14.383 & 0.334 & 15.566 & 0.824 & 0.250 & -1.362 & 1.563 \\
317 & 0.986 & 16.454 & 0.717 & 14.512 & 0.446 & 16.162 & 1.242 & 0.316 & -1.332 & 1.121 \\
353 & 0.599 & 14.515 & 0.394 & 13.248 & 0.258 & 14.361 & 0.801 & 0.352 & -1.440 & 1.336 \\
\hline
\end{tabular}

Notes. Only ten rows are listed here for guidance regarding its form and content. Full table is available at the CDS. 